\title{
Re-examination of the Daily Number of Sunspot Groups for the Royal Observatory, Greenwich (1874-1885)
}

\author{
D.M. Willis ${ }^{1,2}$ - M.N. Wild ${ }^{3} \cdot$ J.S. Warburton ${ }^{3}$
}

Received: 30 September 2015 / Accepted: 27 January 2016 / Published online: 18 February 2016

(c) The Author(s) 2016. This article is published with open access at Springerlink.com

\begin{abstract}
The daily number of sunspot groups on the solar disk, as recorded by the programme of sunspot observations performed under the aegis of the Royal Observatory, Greenwich, UK, and subsequently the Royal Greenwich Observatory (RGO), is re-examined for the interval $1874-1885$. The motivation for this re-examination is the key role that the RGO number of sunspot groups plays in the calculation of Group Sunspot Numbers (Hoyt and Schatten in Solar Phys. 179, 189, 1998a; Solar Phys. 181, 491, 1998b). A new dataset has been derived for the RGO daily number of sunspot groups in the interval $1874-1885$. This new dataset attempts to achieve complete consistency between the sunspot data presented in the three main sections of the RGO publications and also incorporates all known errata and additions. It is argued that days for which no RGO solar photograph was acquired originally should be regarded, without exception, as being days without meaningful sunspot data. The daily number of sunspot groups that Hoyt and Schatten assign to days without RGO photographs is frequently just a lower limit. Moreover, in the absence of a solar photograph, the daily number of sunspot groups is inevitably uncertain because of the known frequent occurrence of sunspot groups that exist for just a single day. The elimination of days without photographs changes the list of inter-comparison days on which both the primary RGO observer and a specified secondary comparison observer saw at least one sunspot group. The resulting changes in the personal correction factors of secondary observers then change the personal correction factors of overlapping tertiary observers, etc. In this way, numerical changes in the personal correction factors of secondary observers propagate away from
\end{abstract}

Sunspot Number Recalibration

Guest Editors: F. Clette, E.W. Cliver, L. Lefèvre, J.M. Vaquero, and L. Svalgaard

$\triangle$ D.M. Willis

david.willis@stfc.ac.uk

1 Space Physics and Operations Division, RAL Space, Science and Technology Facilities Council, Rutherford Appleton Laboratory, Harwell Oxford, Didcot, Oxfordshire OX11 0QX, UK

2 Centre for Fusion, Space and Astrophysics, Department of Physics, University of Warwick, Coventry CV4 7AL, UK

3 UK Solar System Data Centre, RAL Space, Science and Technology Facilities Council, Rutherford Appleton Laboratory, Harwell Oxford, Didcot, Oxfordshire OX11 0QX, UK 
the interval $1874-1885$, thereby potentially changing the arithmetical calculation of Group Sunspot Numbers over an appreciably wider time interval.

Keywords Greenwich photo-heliographic results · Daily number of sunspot groups · Derivation of a new dataset - Days without solar photographs - Sunspot groups that exist for a single day · Temporary invisibility of some sunspot groups · Errors in the calculation of personal correction factors

\section{Introduction}

An accurate measure of the varying levels of solar activity is a prerequisite in a wide range of scientific studies, including investigations of the solar dynamo (Charbonneau, 2010), space weather (Pulkkinen, 2007), space climate (Barnard et al., 2011), solar-terrestrial physics (Lockwood, 2013), global terrestrial climate change (Gray et al., 2010; Lockwood, 2012), and regional terrestrial climate change (Lockwood, 2012). Until almost the start of the twenty-first century, the International Sunspot Number, referred to in the past as the Wolf or Zürich Sunspot Number, was the primary time series used to record the varying levels of solar activity (Clette et al., 2007). Indeed, the International Sunspot Number, which is based on telescopic sunspot observations made by a large number of solar observers over the past four centuries, was for many years the longest quantitative record of solar activity (Waldmeier, 1961; McKinnon, 1987; Clette et al., 2007). At the end of the twentieth century, however, a time series, known as the Group Sunspot Number, was introduced by Hoyt, Schatten, and Nesmes-Ribes (1994), and Hoyt and Schatten (1998a, 1998b). The Hoyt and Schatten reconstruction of past solar activity was designed to be more self-consistent internally (i.e. less dependent upon seeing the tiniest spots) and less noisy than the International Sunspot Number. Moreover, the Hoyt and Schatten time series uses the number of sunspot groups observed, rather than a combination of the number of groups and the number of individual spots. Much attention has been focused recently on attempts to reconcile the discrepancies between the International and Group Sunspot Numbers (Cliver, Clette, and Svalgaard, 2013; Clette et al., 2014; Cliver et al., 2015; Cliver and Ling, 2016).

The purpose of the present article is to re-examine the determination of the daily number of sunspot groups for the Royal Observatory, Greenwich, within the restricted time interval 1874-1885. The programme of sunspot observations conducted under the aegis of the Royal Observatory, Greenwich, UK, which later became the Royal Greenwich Observatory (RGO), plays a key role in the derivation of the Group Sunspot Number for two reasons (Hoyt and Schatten 1998a, 1998b). [The conventional abbreviation RGO is used here to refer both to the Royal Observatory, Greenwich, and to the Royal Greenwich Observatory.] First, the equation for the Group Sunspot Number includes a normalisation number (12.08) that is chosen to make the mean Group Sunspot Number identical to the mean International Sunspot Number (see Section 2.2) for the interval 1874-1976, during which the RGO was acquiring and publishing sunspot data. Second, each individual (or comparison) solar observer's personal correction factor $\left[k^{\prime}\right]$ is chosen to place that observer on the same scale as the RGO (see Section 2.2). For these two reasons alone, there are strong grounds for being meticulously careful about the determination of the daily number of sunspot groups on the solar disk according to the programme of sunspot observations conducted under the aegis of the RGO.

The main difference between this investigation and the one undertaken by Hoyt and Schatten (1998a, 1998b) is that days for which no solar photograph was acquired originally 
are regarded, without exception, as days for which no sunspot data exist. Stated alternatively, no use is made in this investigation of "interpolated" data on days without photographs obtained from photographs acquired on adjoining days. Evidence is presented to show that it is preferable to accept any added "uncertainty" that arises from days without photographs rather than use, for example, lower limits to the number of sunspot groups on such days, especially if comparisons are to be made with the International Sunspot Number. There are many days without photographs at the very beginning of the RGO programme of solar observations, which is the main reason for restricting the present investigation to the interval $1874-1885$.

Another difference between this investigation and the one undertaken by Hoyt and Schatten (1998a, 1998b) arises from the fact that occasionally the RGO publications indicate that a sunspot group was not seen on one or more days within a longer sequence of days of continual observation. If no specific reason is given for such a group not being observed on a particular day within a sequence of consecutive days, such as a poor-quality photograph or a small spot hidden under one of the cross-wires at the principal focus of the corresponding photoheliograph, this group is not included in the count of groups present on that particular day in the present investigation.

\section{The International and Group Sunspot Numbers}

For completeness, it is helpful to define both the International and Group Sunspot Numbers. In addition, further detailed information is provided on the importance of the programme of solar observations conducted under the aegis of the Royal Greenwich Observatory in the derivation of the Group Sunspot Number.

\subsection{The International Sunspot Number $\left(\boldsymbol{R}_{\mathbf{I}}\right)$}

As noted by Cliver, Clette, and Svalgaard (2013), Wolf proposed the following definition of sunspot number in 1856:

$$
R_{\mathrm{I}}=10 G+S,
$$

where $R_{\mathrm{I}}$ is the relative sunspot number (hereafter referred to as the International Sunspot Number), $G$ is the number of sunspot groups, and $S$ is the number of individual spots (counted at a given time on a given day). For historical reasons, the International Sunspot Number $\left[R_{\mathrm{I}}\right]$ has been referred to in the past as the Wolf Sunspot Number $\left[R_{\mathrm{W}}\right]$ and the Zürich Sunspot Number $\left[R_{\mathrm{Z}}\right]$. Observational experience led Wolf to this simple empirical expression, which gives a sunspot group a weight ten times that of an individual spot.

However, because of differences in eyesight, seeing conditions, and telescope aperture and quality, different observers do not necessarily count the same number of spots on a given day (quite apart from the evolution of a spot during the course of a UT day). Similarly, individual observers have to make subjective decisions about what is a single spot (as opposed to a pore) and what is a group of spots. Therefore, Wolf introduced " $k$-factors" to normalise spot counts from other observers to his own counts, which results in a generalisation of Equation (1) to the following form:

$$
R_{\mathrm{I}}=k(10 G+S) .
$$

This last equation has been used to determine the International Sunspot Number over the past four centuries (Waldmeier, 1961; McKinnon, 1987; Clette et al., 2007). Further 
background information on the determination of $R_{\mathrm{I}}$ and its various limitations can be found in the articles by Clette et al. (2007, 2014), and Cliver, Clette, and Svalgaard (2013).

\subsection{The Group Sunspot Number $\left(\boldsymbol{R}_{\mathbf{G}}\right)$}

As noted in the introduction, the only significant revision of the time series representing $R_{\mathrm{I}}$ has been made by Hoyt and Schatten (1998a, 1998b). These authors introduced a daily sunspot index called the Group Sunspot Number $\left[R_{\mathrm{G}}\right]$ defined as follows:

$$
R_{\mathrm{G}}=(12.08 / N) \sum_{i} k_{i}^{\prime} G_{i}
$$

where $G_{i}$ is the daily number of sunspot groups recorded by the $i$ th observer, $k_{i}^{\prime}$ is the $i$ th observer's correction factor, $N$ is the number of observers used to form the daily value, and 12.08 is a normalisation number chosen to make the mean value of $R_{\mathrm{G}}$ equal to the mean value of $R_{\mathrm{I}}$ for the interval $1874-1976$, during which the RGO was acquiring and publishing sunspot data. Hoyt and Schatten (1998a, 1998b) based their index of solar activity solely on the counts of sunspot groups for the following reasons: i) $90 \%$ of the variance in $R_{\mathrm{I}}$ is caused by changes in the number of groups; and ii) many observers specify only the number of groups rather than both the number of groups and the number of individual spots (Hoyt, Schatten, and Nesmes-Ribes, 1994; Hoyt and Schatten 1998a, 1998b).

In the context of the present investigation, it is important to be precise about the definitions of the symbols $N$ and $k_{i}^{\prime}$, and also the normalisation number 12.08, which appear in Equation (3). As already noted, the symbol $N$ simply represents the number of observers used to form the daily value of $R_{\mathrm{G}}$. The normalisation number varies slightly depending on how many observations are used. The current value of 12.08 differs from an earlier value of 11.93, reported previously by Hoyt, Schatten, and Nesmes-Ribes (1994), because of the addition of more than 100,000 sunspot observations since the preliminary study was undertaken. The symbol $k_{i}^{\prime}$ represents the $i$ th observer's correction factor, which is required to place that observer on the same scale as the RGO: the correction factor for the primary RGO "standard observer" is defined to be 1.000. The RGO "standard observer" is Observer Number 332 in Appendix 1 of the article by Hoyt and Schatten (1998a), which lists all 463 observers used in their calculation of Group Sunspot Number. A correction factor for each individual observer is formed by dividing the total number of sunspot groups seen by the RGO "standard observer" by the total number of sunspot groups seen by the comparison observer, limiting the calculation of this ratio to those days for which both observers saw one or more sunspot groups. The quality of the comparison is defined as being the number of inter-comparison days divided by the quantity $\left|1-k_{i}^{\prime}\right|$. Therefore, a high-quality secondary observer is one for whom many comparisons can be made with the primary RGO "standard observer" and whose measurements are most similar to those made by the RGO "standard observer".

The existence of such high-quality secondary observers enables comparisons to be made with tertiary observers, etc., thereby extending the comparisons further back in time to intervals before the commencement of the programme of RGO sunspot observations (Hoyt and Schatten, 1998a). However, if the value of $k_{i}^{\prime}$ for a secondary or a higher-order observer is lower than 0.6 or greater than 1.4, that observer is not used in any inter-comparisons. The value of $k_{i}^{\prime}$ for tertiary observers is found by weighting their ratios to that of the secondary observer by the quality of this second observer. This process is repeated for fourth-, fifth-, sixth-, and seventh-level observers. The technique described maximises the contribution of 
the best and most active observers and minimises the number of intermediate observers between the primary RGO observer and the observer for whom $k_{i}^{\prime}$ is being calculated. It uses all of the information available rather than a selected subset. Finally, because multiple inter-comparison paths are followed, both the mean $k_{i}^{\prime}$ and its standard deviation can be calculated. These values have been tabulated in Appendix 1 of the article by Hoyt and Schatten (1998a).

It is clear from the preceding discussion that the programme of solar observations conducted under the aegis of the RGO, and the resulting publication of the RGO number of sunspot groups on the solar disk for most days in the interval 1874-1976, plays a crucial role in the formal derivation of the daily Group Sunspot Number. The purpose of the present article is to re-examine the derivation of the daily number of sunspot groups for the RGO within the restricted time interval 1874-1885. The main reason for restricting the present study to this interval is that there are many days without photographs at the beginning of the RGO programme of solar observations; after 1885 there are far fewer days per calendar month for which photographs were not acquired. Another reason for restricting this investigation to the interval $1874-1885$ is discussed in the following section.

It should also be noted that Cliver and Ling (2016) found that they could reproduce the personal correction factors $\left[k^{\prime}\right]$ for the solar observers listed by Hoyt and Schatten (1998a: see their Appendix 1) only if they used the interpolated filldata files of observer matrices from the NOAA National Geophysical Data Center website (www.ngdc.noaa.gov/stp/ space-weather/solar-data/solar-indices/sunspot-numbers/group/archive/), rather than the uninterpolated alldata files of observer matrices. As pointed out in this article, however, the situation is complicated further by the fact that the allegedly "un-interpolated" dataset (alldata files) actually contains interpolated or infill data. This initial interpolation or infilling of data occurs mainly on days for which no photograph exists. Consequently, it is doubly important that days for which no RGO photograph was acquired originally should be regarded, without exception, as being days without meaningful sunspot data. Implementing this correction would change the calculation of some personal correction factors, particularly those using sunspot observations during the interval $1874-1885$, thereby changing the arithmetical calculation of Group Sunspot Numbers over a wider time interval. Cliver and Ling (2016) have presented further examples of cases where they were unable to reproduce the personal correction factors determined by Hoyt and Schatten (1998a) if they followed the specific procedures outlined in the articles published by the latter authors.

\section{The Greenwich Photo-heliographic Results}

A summary of the essential background information on the Greenwich Photo-heliographic Results (1874-1976) has been published by Willis et al. (2013). That article provides a comprehensive discussion of the original solar observations, their various applications in scientific research, the format of the different digital datasets, the necessary definitions of the quantities measured, and the initial identification of errors in both the printed publications and the digital datasets. Using this background information, it is possible to define the sources of information used in the present investigation.

\subsection{Greenwich Photo-heliographic Results (1874-1885)}

As noted by Willis et al. (2013), the publication entitled Greenwich Photo-heliographic Results 1874-1885 (Royal Observatory, Greenwich, 1907), which contains supplementary 
results from photographs of the Sun taken at Greenwich (UK), at Harvard College (USA), at Melbourne (Australia), at Dehra Dun (India), and at Pamplemousses (Mauritius), provides a list of Errata and Additions for the years 1877-1885 (pp. xiii to xxiii). The first part of this list (pp. xiii to xviii) provides amendments to the original RGO publications for the years 1877-1885; the second part (pp. xix to xxi) provides amendments to a separate publication by the Solar Physics Committee (1891), which includes additional results for Dehra Dun and Melbourne for the years 1878-1881; and the third part (pp. xxii to xxiii) provides amendments to the subsequent tables (pp. 1-321) in the same publication as the list of Errata and Additions. Therefore, the publication entitled Greenwich Photo-heliographic Results 1874-1885 (Royal Observatory, Greenwich, 1907) provides a later revision and extension of the Greenwich Photo-heliographic Results for the interval 1874-1885, which is another reason for restricting the present study to this early interval.

An intermediate goal of the present study is an attempt to achieve complete consistency, in the RGO daily count of the number of sunspot groups, between the three main sections in the Greenwich Photo-heliographic Results 1874-1885 (Royal Observatory, Greenwich, 1907); namely, i) "Measures of Positions and Areas of Sun Spots and Faculae" (abbreviated to "Measures"); ii) "Ledgers of Areas and Positions of Groups of Sun Spots" (abbreviated to "Ledgers"); and iii) "Total Projected Areas of Sun Spots and Faculae" (abbreviated to "Total Areas"). Implementing the Errata and Additions listed in the Greenwich Photo-heliographic Results 1874-1885 (Royal Observatory, Greenwich, 1907) is a vitally important part of this attempt to achieve consistency in the RGO count of the number of sunspot groups for each day in the interval 1874-1885. Just two of the entries in Table 1 of the article by Erwin et al. (2013) are relevant in this context (namely those for 22 December 1884): the summation errors for the interval 1880-1885 refer to facular areas, not sunspot areas; the single latitude error is irrelevant in the context of the present investigation; and the single day number error is also irrelevant.

Therefore, the RGO publications used in the present investigation are i) the Greenwich Photo-heliographic Results 1874 - 1885 (Royal Observatory, Greenwich, 1907); ii) the separate publication by the Solar Physics Committee (1891); and iii) the annual Greenwich Photo-heliographic Results for the four years 1882, 1883, 1884, and 1885. These latter publications are required to extract the information in the "Measures" sections of the Greenwich Photo-heliographic Results for the interval 1882-1885.

\subsection{The Observatory Codes}

Table 1 of Willis et al. (2013) provides a unique, new, four-letter code for each solar observatory that contributed to the Greenwich Photo-heliographic Results 1874-1976, as well as the (sometimes ambiguous) original codes. Also given in that table are the geographic coordinates and altitude of each solar observatory, together with brief details of the appropriate range of dates and initial estimates of the total number of photographs supplied by each observatory. Table 1 of the present article provides the observatory codes that are relevant to the interval $1874-1885$ in a simplified form. Two further observatory codes have been introduced to ensure that a unique four-letter code, or identifier, can be assigned to every day in the interval 17 April 1874-31 December 1885. The code UNKN is used if a photograph was acquired but the solar observatory is unknown, or uncertain, and the code NONE is used to signify that no photograph was acquired on a specified day.

Very occasionally it is difficult to assign a unique four-letter observatory code (or a unique photograph) to an entry in the Greenwich Photo-heliographic Results 1874-1885 (Royal Observatory, Greenwich, 1907). For example, on p. xxi of the Errata and Additions, it is noted that "The Indian (DHRA) photograph for Nov. 16 [1881] does not show 
Table 1 The solar observatories that contributed to the Greenwich Photo-heliographic Results in the interval 17 April 1874-31 December 1885. Also given are the new four-letter observatory codes (Willis et al., 2013); the number of solar photographs contributed by each known solar observatory (DHRA, GREN, HARV, MAUR, and MELB); the number of solar photographs contributed by unknown, or uncertain, solar observatories (UNKN); and the numbers of days for which no photograph was acquired originally (NONE). It is readily verified that the sum of the numbers in the right-hand column is 4277 , the total number of days in the interval 17 April 1874-31 December 1885.

\begin{tabular}{llc}
\hline Solar observatory & New code & Number of photographs [days] \\
\hline Dehra Dun Observatory, Uttar Pradesh, India & DHRA & 1126 \\
Royal Observatory, Greenwich, London, UK & GREN & 1998 \\
Harvard College Observatory, Cambridge, MA, USA & HARV & 112 \\
Royal Alfred Observatory, Pamplemousses, Mauritius & MAUR & 172 \\
Melbourne Observatory, Victoria, Australia & MELB & 161 \\
Photograph acquired but observatory unknown & UNKN & 52 \\
No photograph acquired & NONE & 656 \\
\hline
\end{tabular}

Groups 601 and 608, which are seen on the Greenwich (GREN) photograph for that date. The Greenwich measures for those two groups have therefore been adopted in the formation of the Ledgers in the present volume; increasing the Total Area for Whole Spots in line 29, from 1105 to 1117 [p. 73, column 1, line 29 of the Measures (Solar Physics Committee, 1891)]". However, the Ledgers in the Greenwich Photo-heliographic Results 1874-1885 (Royal Observatory, Greenwich, 1907) give GREN for just Group 608 but give DHRA for Group 601 and all the other Groups that existed on 16 November 1881 (603, 605, 606, and 607). The code DHRA is retained in this investigation because this is the code associated with all but one of the sunspot groups that were present on the solar disk on 16 November 1881 .

The code UNKN is used if it is certain that a photograph was acquired but the solar observatory is not identified explicitly. For example, it is noted on p. xxii of the list of Errata and Additions in the Greenwich Photo-heliographic Results 1874-1885 (Royal Observatory, Greenwich, 1907) that "Photographs taken on the following additional dates show neither Spots nor Faculae". This statement is followed by a list of 59 dates for the consecutive years 1874 (10), 1875 (3), 1876 (11), and 1877 (35). However, the solar observatory is not indicated explicitly. Furthermore, the official RGO Archives at the University of Cambridge Library for this four-year interval (MSS.RGO.51/6 - 235; janus.lib.cam.ac.uk/db/node.xsp?id=EAD/GBR/0180/RGO 51) do not contain solar plates' contact prints for any of these 59 dates (i.e. the 59 dates are not among the 230 dates cited within MSS.RGO.51/6 - 235). Presumably this is because no spots or faculae were observed on these days and hence any contact prints made from the solar plates were not archived. The Introduction (pp. v to xii) to the Greenwich Photo-heliographic Results 1874-1885 (Royal Observatory, Greenwich, 1907) contains tables giving the annual number of photographs contributed by each solar observatory for the years 1874 to 1885 ; these numbers were used to compile the entries in Table 1 of Willis et al. (2013).

In general, however, it may not be possible to attribute unambiguously the 59 additional dates (for which the photographs show neither spots nor faculae) to specific solar observatories. A clear exception to this last statement is the first year of the interval 1874-1887. In 1874, the total numbers of photographs taken at Greenwich (GREN) and Harvard (HARV), respectively, are cited as being 139 and 2. Since the two photographs from Harvard ( 9 and 12 December 1874) both show sunspots, the ten additional photographs in 1874 (which show 
neither spots nor faculae) can be attributed unambiguously to Greenwich (GREN). It is not immediately obvious that similar arguments can be adduced for the years 1875,1876 , and 1877. Furthermore, a preliminary check suggests that the annual numbers of photographs assigned to the various solar observatories for the years 1875,1876 , and 1877, in the Introduction to the Greenwich Photo-heliographic Results 1874-1885 (Royal Observatory, Greenwich, 1907), may need to be revised. Further research is required to resolve this matter. In the present investigation the code UNKN is always assigned if the solar observatory is uncertain or unknown.

There are only three further uncertainties in the provenance of the solar photographs in the interval 17 April 1874-31 December 1885. For 19 March 1879, the "Measures" section of the report by the Solar Physics Committee (1891) indicates that the photograph was acquired at Dehra Dun (DHRA), whereas the list of spotless days at the end of the report indicates that the photograph was acquired at Greenwich (GREN). There is no entry in the "Ledgers" section of the Greenwich Photo-heliographic Results 1874-1885 (Royal Observatory, Greenwich, 1907). The official RGO Archives at the University of Cambridge Library for the year 1879 (MSS.RG0.51/250 - 276; janus.lib.cam.ac.uk/db/node.xsp?id=EAD/GBR/0180/RGO 51) do not contain a contact print for 19 March 1879, again presumably because no spots were observed on this day. Therefore, the solar observatory is essentially unknown on this day. Similar unresolvable inconsistencies in identifying the solar observatory at which the solar photograph was acquired occur on 15 August 1881 and 16 August 1881 (see also p. xxi of the Errata and Additions for 16 August). Hence it is assumed the solar observatory is unknown (UNKN) in these three cases.

The code NONE normally corresponds to the entry 'No Photograph' in the "Total Areas" section of the Greenwich Photo-heliographic Results 1874-1885 (Royal Observatory, Greenwich, 1907), which is often (but not always) confirmed by the entry 'No photograph' (NP in the dataset) in the "Ledgers" section of this same publication. However, it is noted on p. xix of the Errata and Additions in the Greenwich Photo-heliographic Results 1874-1885 (Royal Observatory, Greenwich, 1907) that the photograph acquired at Dehra Dun (DHRA) on 29 January 1880 "has not been used for the Ledger, or for the computation of Projected Areas in the present Volume", although no reason is given. Hence the code NONE is also assigned to 29 January 1880 in this investigation.

\section{New Dataset for the RGO Numbers of Sunspot Groups}

Figure 1 illustrates the "boundaries" of the various sunspot groups and solar faculae observed on the solar photograph acquired at the Royal Observatory, Cape of Good Hope, South Africa, on 26 February 1918 (MS.RGO.50/57; janus.lib.cam.ac.uk/db/node.xsp?id= EAD/GBR/0180/RGO 50), as delineated by staff at the Royal Observatory, Greenwich. Despite the fact that Figure 1 relates to a date long after the time interval being investigated (1874-1885), it illustrates the general procedure adopted at the outset. In Figure 1 the boundary contours of sunspot groups are delineated and numbered, and the groups themselves are also numbered. The explanatory text describing the archive collection of Royal Observatory (RO) and Royal Greenwich Observatory (RGO) Solar Plates' Contact Prints (janus.lib.cam.ac.uk/db/node.xsp?id=EAD/GBR/0180/RGO 51), stored in the Cambridge University Library, contains the following statement: "From the early 20th century the prints show the hand-drawn circles, arrows and reference codes made on the glass-plate negatives to mark sun spots and faculae". In the first decade of the twentieth 
Figure 1 The boundaries of the various sunspot groups and solar faculae observed on the solar photograph acquired at the Cape of Good Hope on 26 February 1918 (MS.RGO.50/57), as delineated by staff at the Royal Observatory, Greenwich. Although this photograph relates to a date long after the time interval considered (1874-1885), it illustrates the numbering system adopted at the outset by the RGO staff. The boundary contours of sunspot groups are delineated and numbered, and the groups themselves are also numbered. (Figure 1 is reproduced by kind permission of the Syndics of Cambridge University Library.)

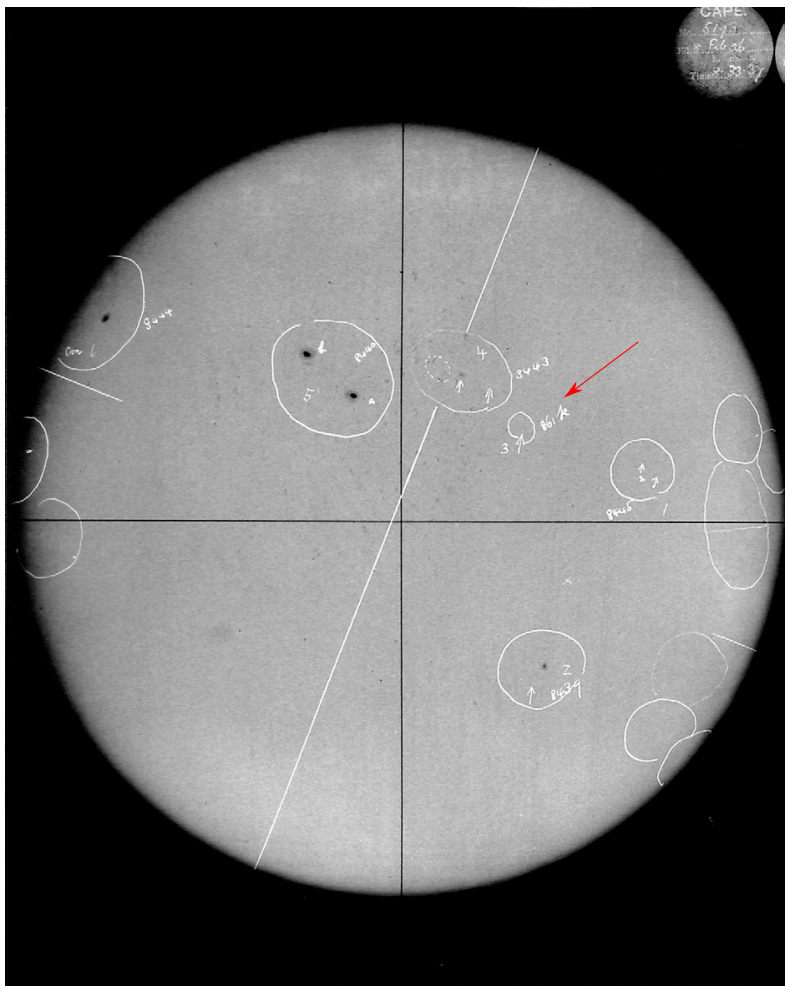

century, it appears that the boundaries of sunspot groups were delineated but not numbered. A.J. Perkins (Curator of Scientific Manuscripts, Cambridge University Library) very kindly looked through all 226 surviving solar plates' contact prints for 1884, the penultimate year of the interval 1874-1885 (A.J. Perkins, Private communication, 2014). He concluded that there were meaningful marks on just two plates (namely MS.RGO.51/928 and MS.RGO.51/948) corresponding to 06 and 28 January 1884, respectively. These marks seemed to be intentional if crude. However, there was no evidence of marking of the plates in any consistent and purposeful manner in 1884.

Although the solar photographs were acquired at the various observatories listed in Table 1, the "boundaries" of the sunspot groups were always delineated by the RGO staff. Each distinct sunspot group was assigned a unique number, as illustrated in Figure 1, which it retained throughout its passage across the solar disk. The first sunspot group recorded in the Greenwich Photo-heliographic Results 1874 - 1885 (Royal Observatory, Greenwich, 1907) - on 17 April 1874 - was assigned the number 82. The number 1 was assigned to one of the four sunspot groups (Numbers 1, 2, 3, and 4) present on the solar disk on 21 July 1873, although the format of the data before 17 April 1874 (Greenwich Astronomical Results, 1874) is slightly different to that published subsequently in the Greenwich Photo-heliographic Results 1874-1976. The number of distinct sunspot groups on a solar photograph, which is denoted by the symbol $G$ in Equation (3), is then just the count of the numbered sunspot groups on that particular day. Although the boundaries of the sunspot groups, and hence the number of distinct sunspot groups, are somewhat subjective, the present investigation is based solely on information presented in the RGO publications listed in Section 3.1, in- 


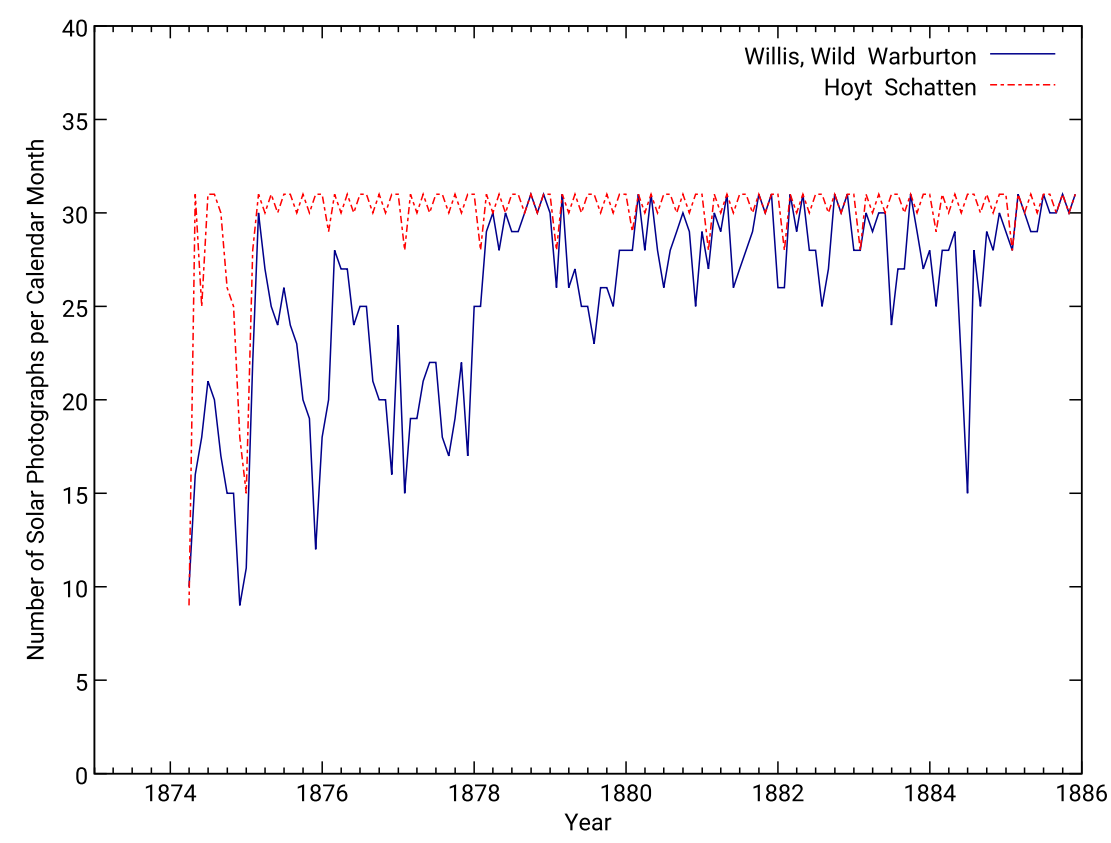

Figure 2 The number of days per calendar month, within the interval 17 April 1874-31 December 1885, for which RGO solar photographs were acquired. The solid blue line is a graphical representation of the information in the new dataset derived in this investigation and the broken red line represents the corresponding information extracted from the work of Hoyt and Schatten (1998a, 1998b).

cluding the Errata and Additions in the Greenwich Photo-heliographic Results 1874-1885 (Royal Observatory, Greenwich, 1907).

Particular attention is drawn to the sunspot group numbered $861 \mathrm{k}$ in Figure 1, which is identified by a red arrow. This is the 11th, and last, sunspot group that existed for one day only during Carrington Rotation 861: the order of appearance of such sunspot groups is indicated by a lower-case letter ( $\mathrm{a}-\mathrm{k}$ in the case of Carrington Rotation 861). Commencing at the beginning of 1916, this separate system of numbering sunspot groups was used for groups that existed for just a single day. Before 1916, sunspot groups that existed for a single day were numbered in exactly the same sequence as sunspot groups that existed for longer than one day. From the beginning of 1959, a numerical prefix or suffix was used instead of a lower-case letter suffix. A two-digit numerical suffix is used in the digital datasets (Willis et al., 2013; Section 5). Furthermore, it should be noted that the Royal Greenwich Observatory also published separate general catalogues of sunspots seen on one day only throughout the interval 1933-1976. The significance of sunspot groups that existed for just one day during the interval $1874-1885$ is discussed further in Section 4.3.

\subsection{Format of the New Dataset}

The intention in this subsection is to define the format of the new dataset by presenting a few examples of the appropriate information for a sequence of consecutive days; these particular examples illustrate the main differences between the present investigation and the one undertaken by Hoyt and Schatten (1998a, 1998b). 


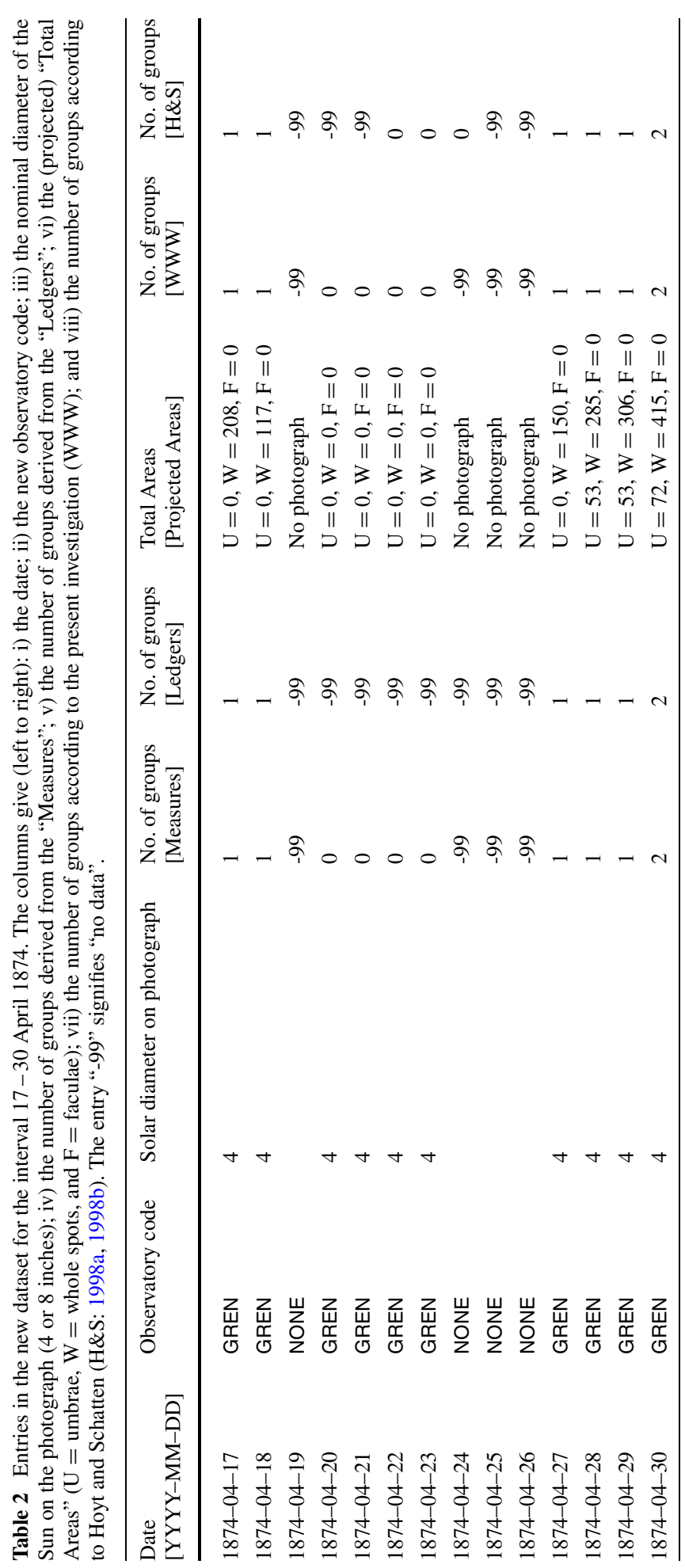




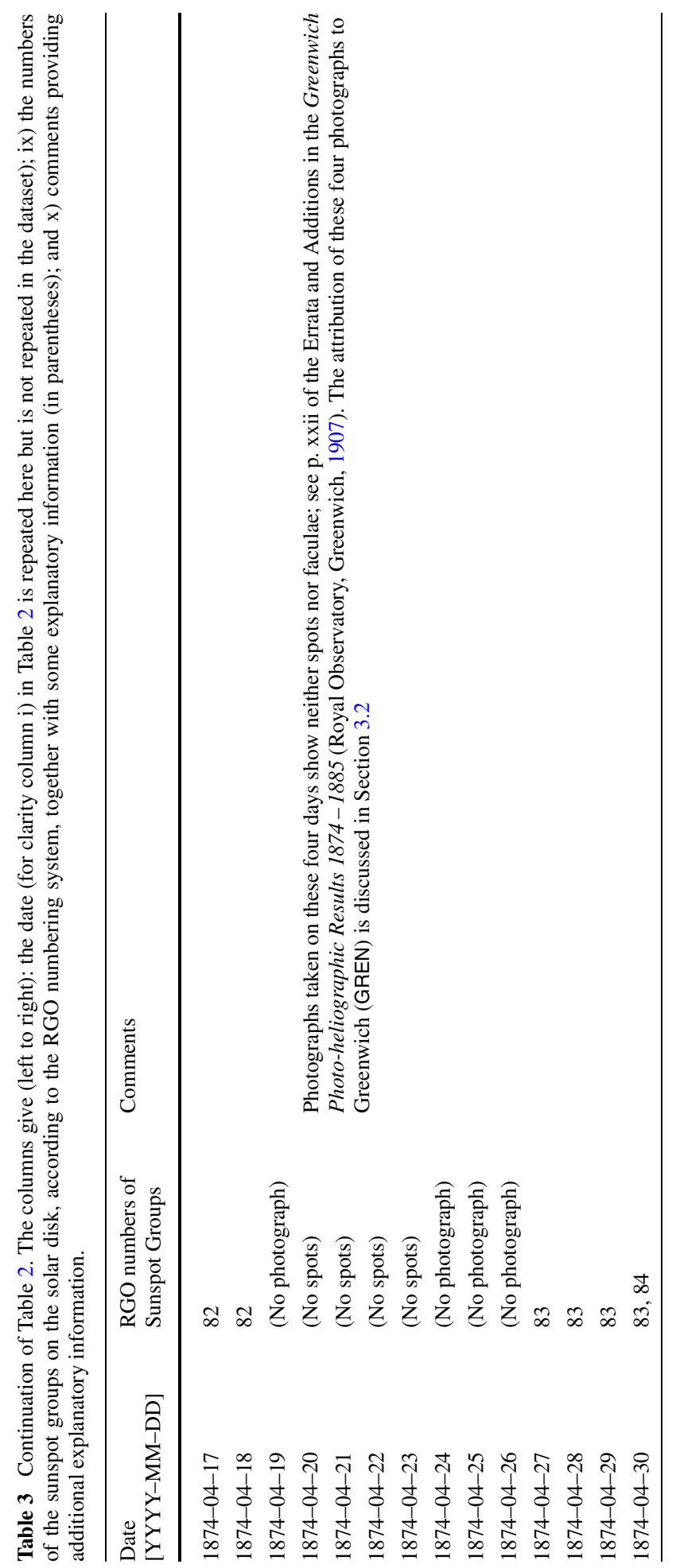




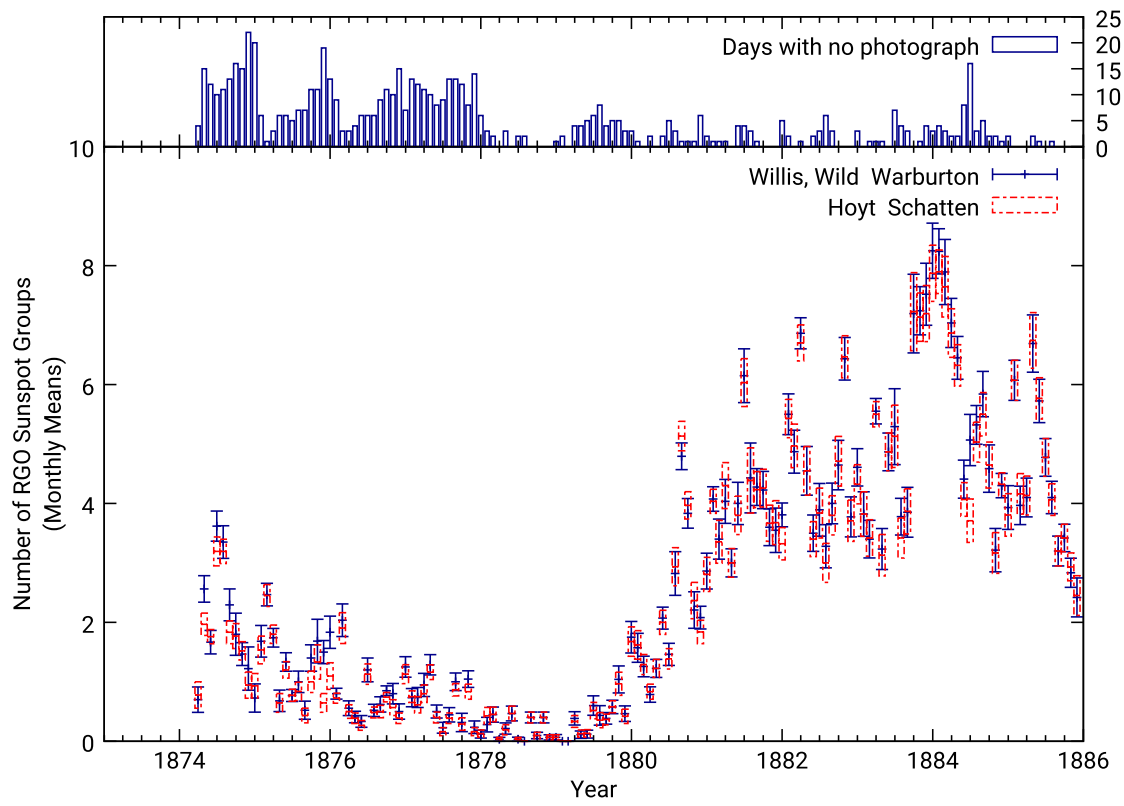

Figure 3 The monthly means of the RGO number of sunspot groups and the standard errors of these monthly means for the interval 17 April 1874-31 December 1885. As in the case of Figure 2, results presented in blue (solid error bars) are from the present investigation and results presented in red (broken candlesticks) are from the work of Hoyt and Schatten (1998a, 1998b). The histogram at the top of this figure shows the number of days per calendar month without solar photographs according to the present investigation.

For example, Tables 2 and 3 present the entries in the new dataset for the interval 17 30 April 1874, which constitutes the first 14 days of this dataset. The columns in Table 2 give (left to right) i) the date; ii) the new observatory code; iii) the nominal diameter of the Sun on the photograph (four or eight inches); iv) the number of groups derived from the "Measures"; v) the number of groups derived from the "Ledgers"; vi) the "Total Areas" (i.e. the projected umbral (U), whole-spot (W) and facular (F) areas); vii) the number of groups according to the present investigation (WWW); and viii) the number of groups according to Hoyt and Schatten (H\&S). The columns in Table 3, which is a continuation of Table 2, give (left to right) the date (for clarity, column i) in Table 2 is repeated in Table 3 but is not repeated in the dataset itself); ix) the numbers of the sunspot groups on the solar disk, according to the RGO numbering system, together with some explanatory information (in parentheses); and (x) comments providing additional explanatory information.

It is clear from Tables 2 and 3 that the discrepancies between the present results and those derived by Hoyt and Schatten (1998a, 1998b) occur either when no photograph was available originally or when additional information extracted from the Errata and Additions in the Greenwich Photo-heliographic Results 1874 - 1885 (Royal Observatory, Greenwich, 1907) is incorporated. However, there is no one-to-one correspondence in the interval 17-30 April 1874 either between discrepancies and missing photographs, or between discrepancies and additional information.

Tables 4 and 5 present the entries in the new dataset for the 14-day interval 18-31 July 1884. It is clear from these tables that the main differences between the present results and those published by Hoyt and Schatten (1998a, 1998b) occur on days when no photograph is available. For example, since the Sunspot Groups 1436, 1437, and 1438 are seen on the 


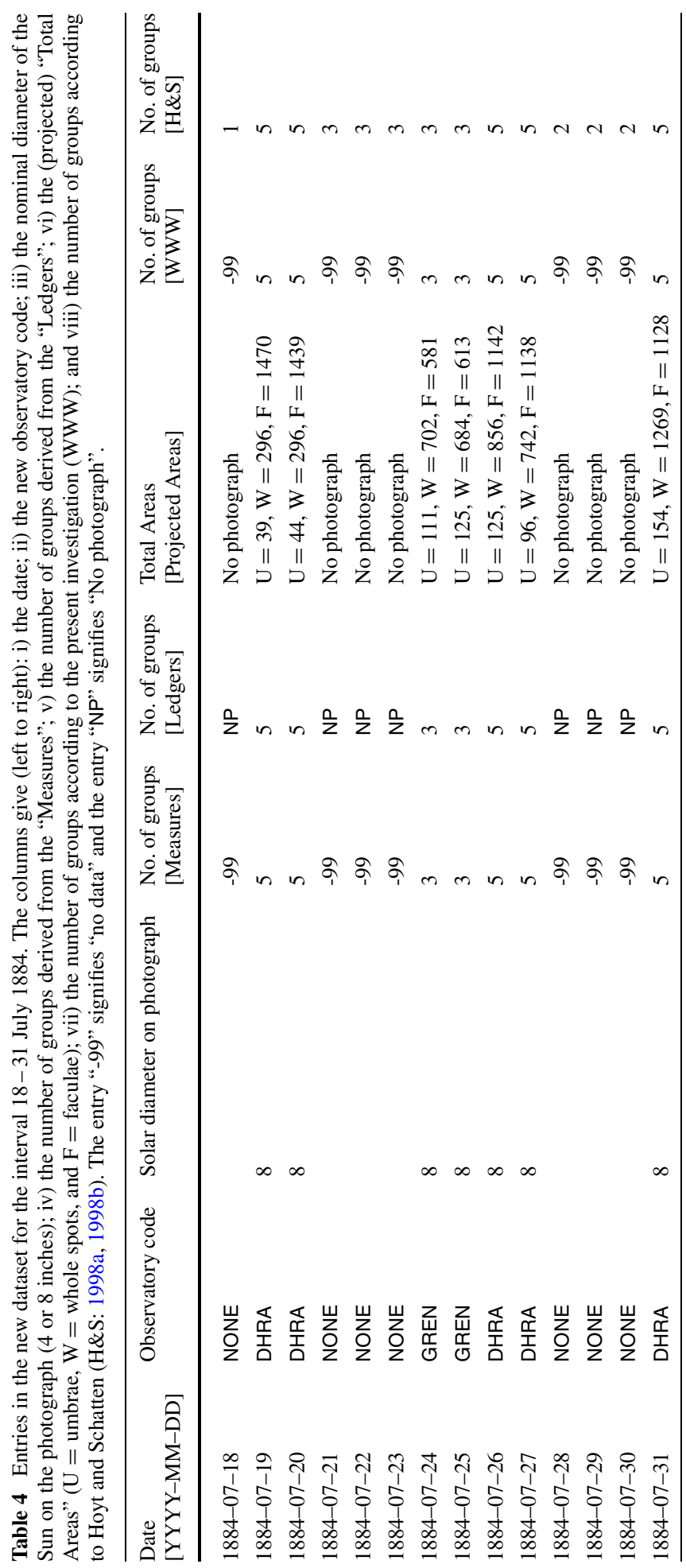




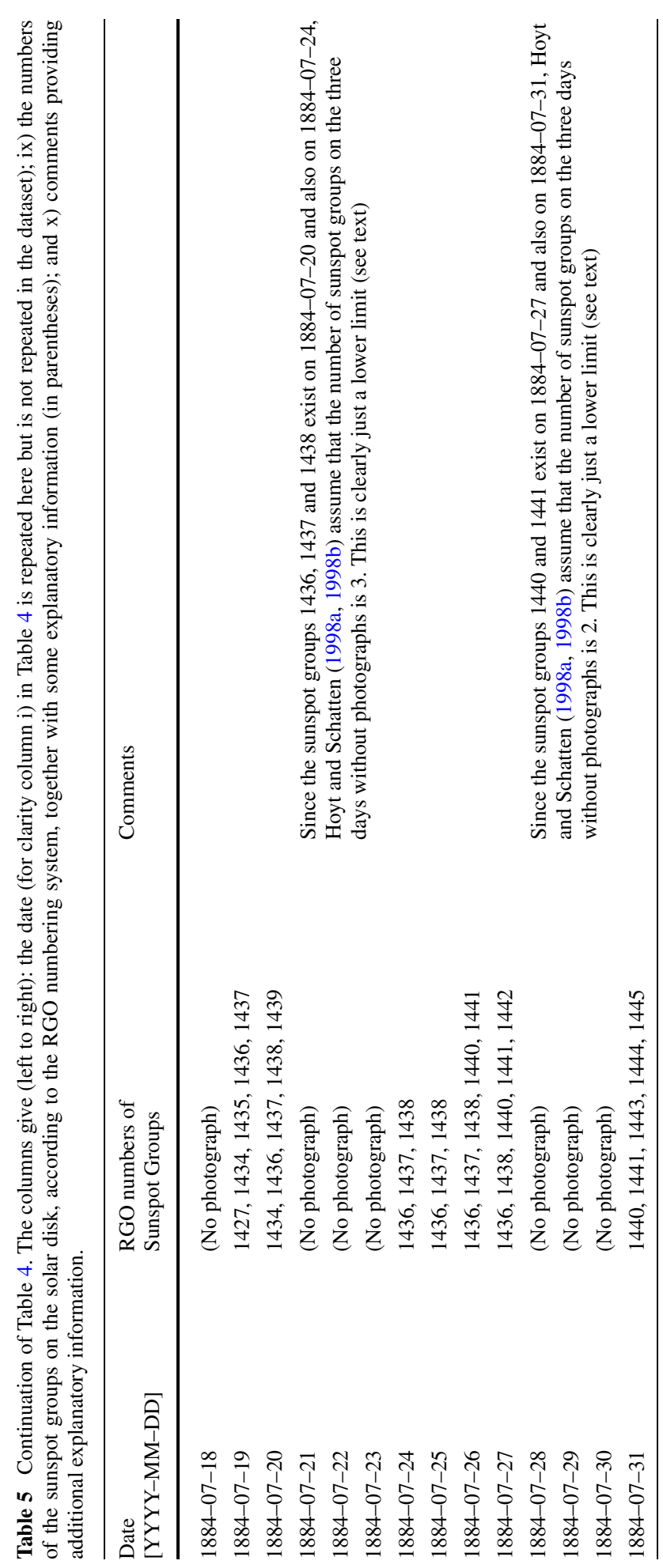


solar disk on both 20 July and 24 July, Hoyt and Schatten assume that the number of sunspot groups that exist on the three intervening days without photographs is three. However, this is clearly just a lower limit. In particular, in the absence of photographs, it is not known if either of the Sunspot Groups 1434 and 1439 persisted for a further one, two, or three days after 20 July. Similarly, since the Sunspot Groups 1440 and 1441 are seen on the solar disk on both 27 July and 31 July, Hoyt and Schatten assume that the number of sunspot groups that exist on the three intervening days without photographs is two. Once again, this is just a lower limit. In this second case, however, it is not known if any of the Sunspot Groups 1436, 1438, and 1442 persisted for a further one, two, or three days after 27 July, and it is also not known if any of the Sunspot Groups 1443, 1444, and 1445 appeared one, two, or three days before 31 July. The examples presented in Tables 4 and 5 are definitely not rare occurrences; there are numerous similar examples in the interval 1874-1885. Conversely, there are many situations in which Hoyt and Schatten (1998a, 1998b) probably assigned to days without photographs the correct number of sunspots on the solar disk.

As an added complication, however, there are many examples of sunspots that existed for just a single day, as illustrated in Figure 1 for 26 February 1918 (Group 861k, identified by the red arrow) and indicated in Table 10 for the interval 1874-1885 (see Section 4.3). Therefore, one or more sunspots that existed for just a single day could have been present on the solar disk on any day for which no photograph is available. Consequently, it is preferable to assume that no information is available on days without photographs.

Tables 6 and 7, which present data from the new dataset for the interval 18-31 October 1882 , illustrate a rather more subtle difference between the results of this investigation and those published by Hoyt and Schatten (1998a, 1998b). It should be noted that there are no days without photographs in this 14-day interval. However, the information in the Ledgers indicates that both the umbral (U) and whole-spot (W) areas are zero for Group Number 865 on 24 October 1882. Similarly, both the umbral and whole-spot areas are zero for Group Number 871 on 31 October 1882 (and on 1 November 1882). No explanation is given in the RGO publications for these areas being zero on one, or more, days within a sequence of days for which the sunspot group has non-zero areas on the other days. This difference between the present results and those published by Hoyt and Schatten (1998a, 1998b) is discussed further in Section 4.4.

\subsection{Availability of Solar Photographs}

Table 8 indicates the total number of solar photographs available for each calendar year of the interval 17 April 1874-31 December 1885. This information has been gleaned from the Introduction to the Greenwich Photo-heliographic Results 1874-1885 (Royal Observatory, Greenwich, 1907). Also listed are the number of days in each calendar year and the percentage of days for which photographs exist (accurate to the nearest integer value). The annual number of available photographs tends to increase as time progresses within the interval $1874-1885$, although this increase is not monotonic. It should be noted that there are as many as 51 days without photographs in the penultimate year. For comparison, the annual number of solar photographs available in the following ten-year interval 1886-1895 (and the percentage coverage to the nearest integer value) are as follows: $363(99 \%), 361(99 \%)$, $359(98 \%), 360(99 \%), 361$ (99\%), 363 (99\%), 362 (99\%), 362 (99\%), $364(100 \%)$, and $364(100 \%)$. These values have been derived from the "Total Projected Areas" sections of the Greenwich Photo-heliographic Results for the individual years 1886 to 1895 . Therefore, it is the first 12-year interval $1874-1885$, especially the early part of this interval, that is critically important in terms of missing photographs. 


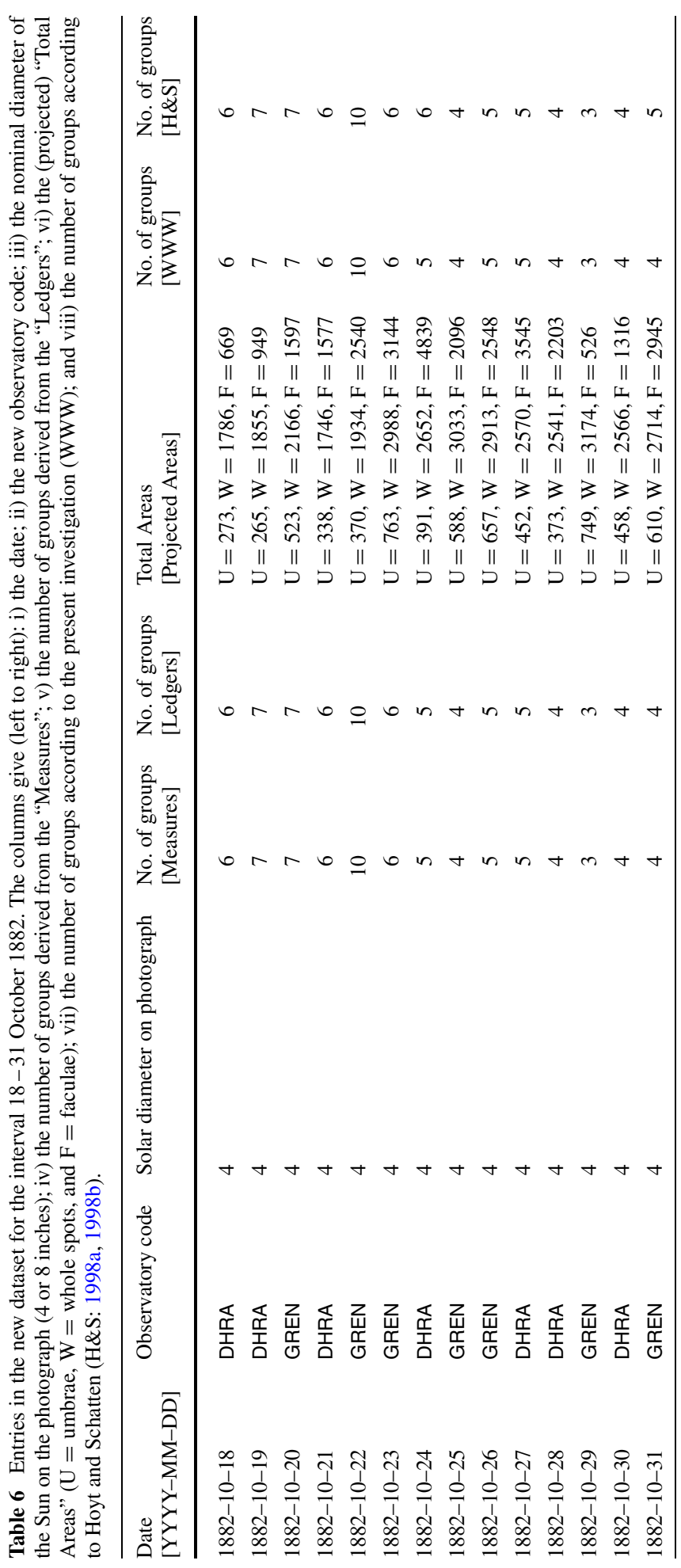


Table 7 Continuation of Table 6. The columns give (left to right): the date (for clarity column i) in Table 6 is repeated here but is not repeated in the dataset); ix) the numbers of the sunspot groups on the solar disk, according to the RGO numbering system; and $\mathrm{x}$ ) comments providing additional explanatory information.

\begin{tabular}{lll}
\hline $\begin{array}{l}\text { Date } \\
\text { [YYYY-MM-DD] }\end{array}$ & RGO numbers of Sunspot Groups & Comments \\
\hline
\end{tabular}

$\begin{array}{ll}1882-10-18 & 856,857,858,859,860,861 \\ 1882-10-19 & 856,857,859,860,861,862,863 \\ 1882-10-20 & 856,857,859,861,862,863,864 \\ 1882-10-21 & 856,857,859,861,862,864 \\ 1882-10-22 & 856,857,859,861,862,864,865,866,867,868 \\ 1882-10-23 & 857,862,864,865,866,868 \\ 1882-10-24 & 857,862,864,866,868\end{array}$

$\begin{array}{ll}1882-10-25 & 864,865,866,868 \\ 1882-10-26 & 864,865,866,868,869 \\ 1882-10-27 & 864,865,868,869,870 \\ 1882-10-28 & 864,868,869,870 \\ 1882-10-29 & 868,870,871 \\ 1882-10-30 & 868,870,871,872 \\ 1882-10-31 & 868,870,872,873\end{array}$

According to the Ledgers, $\mathrm{U}=\mathrm{W}=0$ for Gr. No. 865 on October 24. No reason is given
According to the Ledgers, $\mathrm{U}=\mathrm{W}=0$ for Gr. No. 871 on October 31. No reason is given

Table 8 The total number of solar photographs available for each calendar year of the interval 17 April $1874-31$ December 1885 .

\begin{tabular}{llll}
\hline Year & Number of photographs & Number of days in year & Percentage coverage [\%] \\
\hline 1874 & 141 & 259 & 54 \\
1875 & 263 & 365 & 72 \\
1876 & 271 & 366 & 74 \\
1877 & 235 & 365 & 64 \\
1878 & 347 & 365 & 95 \\
1879 & 318 & 365 & 87 \\
1880 & 341 & 366 & 93 \\
1881 & 348 & 365 & 95 \\
1882 & 343 & 365 & 94 \\
1883 & 340 & 365 & 93 \\
1884 & 315 & 366 & 86 \\
1885 & 359 & 365 & 98 \\
\hline
\end{tabular}

Table 9 shows the number of days per calendar month, within the interval 17 April $1874-$ 31 December 1885, for which no solar photograph was acquired. The numbers in this table have been derived directly from the dataset. It should be noted that the annual numbers 
Table 9 Number of days per calendar month, within the interval 17 April 1874-31 December 1885, for which no solar photograph was acquired.

\begin{tabular}{|c|c|c|c|c|c|c|c|c|c|c|c|c|c|}
\hline \multirow[t]{2}{*}{ Year } & \multicolumn{12}{|c|}{ Month } & \multirow[t]{2}{*}{ Total } \\
\hline & Jan & $\mathrm{Feb}$ & Mar & Apr & May & Jun & Jul & Aug & Sep & Oct & Nov & Dec & \\
\hline 1874 & - & - & - & 4 & 15 & 12 & 10 & 11 & 13 & 16 & 15 & 22 & 118 \\
\hline 1875 & 20 & 6 & 1 & 3 & 6 & 6 & 5 & 7 & 7 & 11 & 11 & 19 & 102 \\
\hline 1876 & 13 & 9 & 3 & 3 & 4 & 6 & 6 & 6 & 9 & 11 & 10 & 15 & 95 \\
\hline 1877 & 7 & 13 & 12 & 11 & 10 & 8 & 9 & 13 & 13 & 12 & 8 & 14 & 130 \\
\hline 1878 & 6 & 3 & 2 & 0 & 3 & 0 & 2 & 2 & 0 & 0 & 0 & 0 & 18 \\
\hline 1879 & 1 & 2 & 0 & 4 & 4 & 5 & 6 & 8 & 4 & 5 & 5 & 3 & 47 \\
\hline 1880 & 3 & 1 & 0 & 2 & 0 & 2 & 5 & 3 & 1 & 1 & 1 & 6 & 25 \\
\hline 1881 & 2 & 1 & 1 & 1 & 0 & 4 & 4 & 3 & 1 & 0 & 0 & 0 & 17 \\
\hline 1882 & 5 & 2 & 0 & 1 & 0 & 2 & 3 & 6 & 3 & 0 & 0 & 0 & 22 \\
\hline 1883 & 3 & 0 & 1 & 1 & 1 & 0 & 7 & 4 & 3 & 0 & 1 & 4 & 25 \\
\hline 1884 & 3 & 4 & 3 & 2 & 2 & 8 & 16 & 3 & 5 & 2 & 2 & 1 & 51 \\
\hline 1885 & 2 & 0 & 0 & 0 & 2 & 1 & 0 & 1 & 0 & 0 & 0 & 0 & 6 \\
\hline Total & 65 & 41 & 23 & 32 & 47 & 54 & 73 & 67 & 59 & 58 & 53 & 84 & 656 \\
\hline
\end{tabular}

of days without photographs (column 14 of Table 9) agree exactly with the corresponding numbers ("Days without record") in the Introduction to the Greenwich Photo-heliographic Results 1874-1885 (Royal Observatory, Greenwich, 1907), which provides an important consistency check on the dataset. It is clear from Table 9 that there are many days without photographs in the initial four-year interval 1874-1877. Thereafter the numbers of days without photographs decreases, although there are 51 days without photographs in 1884 . Indeed, the fact that there are 16 days without photographs in July 1884 accounts for the discrepancies between the results of this investigation and that published by Hoyt and Schatten (1998a, 1998b) shown in Tables 4 and 5.

\subsection{Sunspot Groups that Existed for a Single Day}

As noted in the introductory part of Section 4, there are numerous examples throughout the entire interval $1874-1976$ of sunspots that existed for just a single day. Table 10 presents the number of occurrences per calendar month, within the interval 17 April 1874-31 December 1885 , of a sunspot group being seen on just a single day. In order to define rigorously a sunspot group that existed for just a single day, it must be absent on photographs acquired on both the immediately preceding and following days. Since there are many days without a photograph in the interval $1874-1877$, as indicated in Table 9, the numbers presented in Table 10 for this four-year interval are gross underestimates, which do not represent the true situation. However, there are fewer days without photographs in the interval 1880-1885 (see Table 9), so the numbers in Table 10 for this six-year interval are more likely to be representative of the true situation. On average, sunspots that existed for just a single day occurred about four times per calendar month. Therefore, sunspots that existed for just a single day could have been present on the solar disk on a number of the days for which solar photographs are unavailable. This conclusion strengthens the argument for assuming that no meaningful information is available on days without photographs, instead of following the procedure adopted by Hoyt and Schatten (1998a, 1998b), who used "interpolated data" on many of the days without photographs. 
Table 10 Number of occurrences per calendar month, within the interval 17 April 1874-31 December 1885, of a sunspot group being seen on just a single day. In order to define the existence of a sunspot group for just a single day, it must be absent on photographs acquired on both the immediately preceding and following days.

\begin{tabular}{|c|c|c|c|c|c|c|c|c|c|c|c|c|c|}
\hline \multirow[t]{2}{*}{ Year } & \multicolumn{12}{|c|}{ Month } & \multirow[t]{2}{*}{ Total } \\
\hline & Jan & Feb & Mar & Apr & May & Jun & Jul & Aug & Sep & Oct & Nov & Dec & \\
\hline 1874 & - & - & - & 0 & 0 & 0 & 0 & 0 & 0 & 0 & 0 & 0 & 0 \\
\hline 1875 & 1 & 0 & 1 & 0 & 1 & 1 & 1 & 1 & 1 & 0 & 0 & 0 & 7 \\
\hline 1876 & 0 & 0 & 0 & 3 & 0 & 0 & 0 & 0 & 0 & 0 & 1 & 0 & 4 \\
\hline 1877 & 0 & 0 & 0 & 0 & 0 & 0 & 0 & 0 & 1 & 0 & 0 & 0 & 1 \\
\hline 1878 & 0 & 2 & 0 & 1 & 0 & 1 & 1 & 0 & 0 & 0 & 0 & 0 & 5 \\
\hline 1879 & 0 & 0 & 0 & 1 & 0 & 0 & 0 & 0 & 0 & 0 & 1 & 0 & 2 \\
\hline 1880 & 4 & 1 & 0 & 1 & 3 & 1 & 1 & 3 & 1 & 8 & 8 & 0 & 31 \\
\hline 1881 & 2 & 5 & 6 & 6 & 8 & 2 & 5 & 5 & 2 & 3 & 9 & 5 & 58 \\
\hline 1882 & 2 & 9 & 6 & 4 & 6 & 7 & 2 & 3 & 2 & 1 & 4 & 6 & 52 \\
\hline 1883 & 8 & 6 & 1 & 4 & 9 & 7 & 3 & 2 & 3 & 4 & 4 & 4 & 55 \\
\hline 1884 & 7 & 2 & 9 & 6 & 4 & 1 & 2 & 3 & 4 & 5 & 4 & 2 & 49 \\
\hline 1885 & 3 & 3 & 4 & 5 & 5 & 3 & 4 & 6 & 3 & 2 & 2 & 6 & 46 \\
\hline Total & 27 & 28 & 27 & 31 & 36 & 23 & 19 & 23 & 17 & 23 & 33 & 23 & 310 \\
\hline
\end{tabular}

For completeness, the RGO numbers of the sunspot groups in the interval 17 April $1874-$ 31 December 1885 that existed for just one day are listed in Appendix A, together with their dates of occurrence.

\subsection{Temporary Invisibility of Some Sunspot Groups}

As noted in the Introduction and Section 4.1 ( $c f$. Tables 6 and 7), the RGO publications occasionally indicate that a sunspot group was not seen on one or more days within a longer sequence of days of continual observation. For example, Table 11 presents the Ledger for RGO Group Number 149*. This table and the footnote are reproduced, with minor changes, from the Greenwich Photo-heliographic Results 1874-1885 (Royal Observatory, Greenwich, 1907). The table is taken from the Ledgers and the footnote is taken from the Measures, although the footnote is repeated (apart from the date range) immediately before the table in the Ledgers. It is clear from Table 11 that Group Number 149* was apparently present on the solar disk during the seven-day interval 10-16 March 1875, but the recorded umbral and whole-spot areas are both zero on 15 March. No explanation is given for Group Number 149* being invisible on 15 March. Likewise, Table 12 presents the Ledger for RGO Group Number 365. This table is also reproduced, with minor changes, from the Greenwich Photo-heliographic Results 1874 - 1885 (Royal Observatory, Greenwich, 1907). In this case, Group Number 365 was apparently present on the solar disk during the seven-day interval 20-26 September 1880, but the recorded umbral and whole-spot areas are both zero throughout the four-day interval $21-24$ September. Once again, no explanation is given for Group Number 365 being invisible in this four-day interval. The example presented in Table 12 is not an extreme case. Group Number 454 was apparently present on the solar disk during the 12-day interval 8-19 April 1881, but the recorded umbral and whole-spot areas are both zero throughout the eight-day interval 10-17 April. In such cases, the verbal description immediately before the table confirms that the sunspot group was not seen on days for which the umbral and whole-spot areas are both zero. 


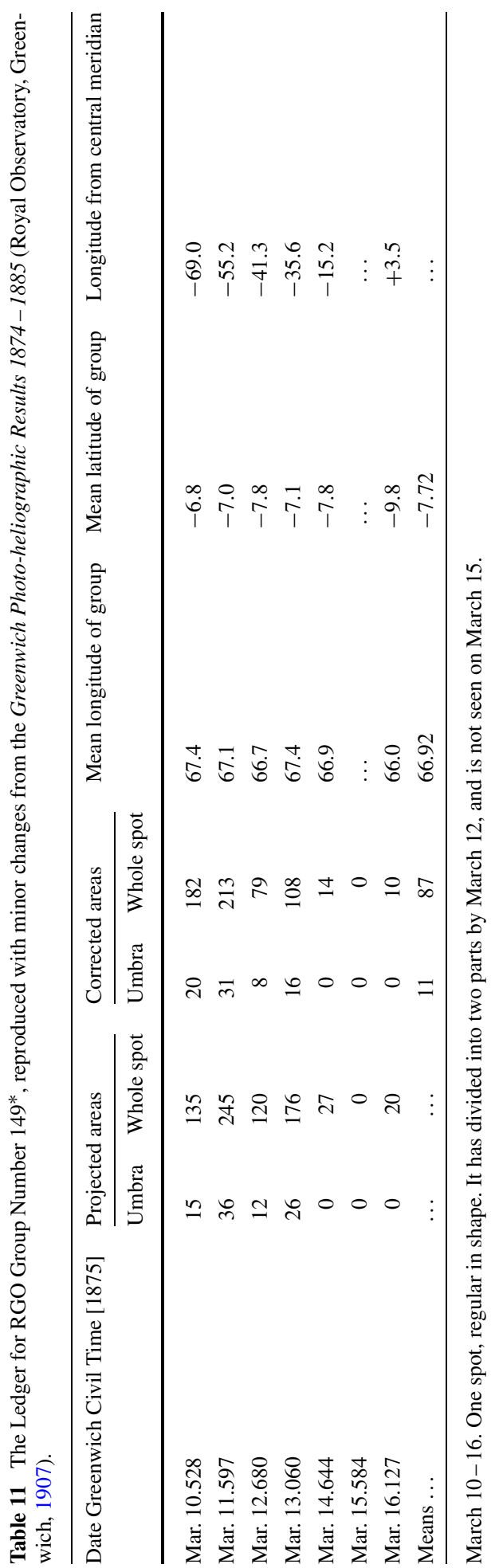




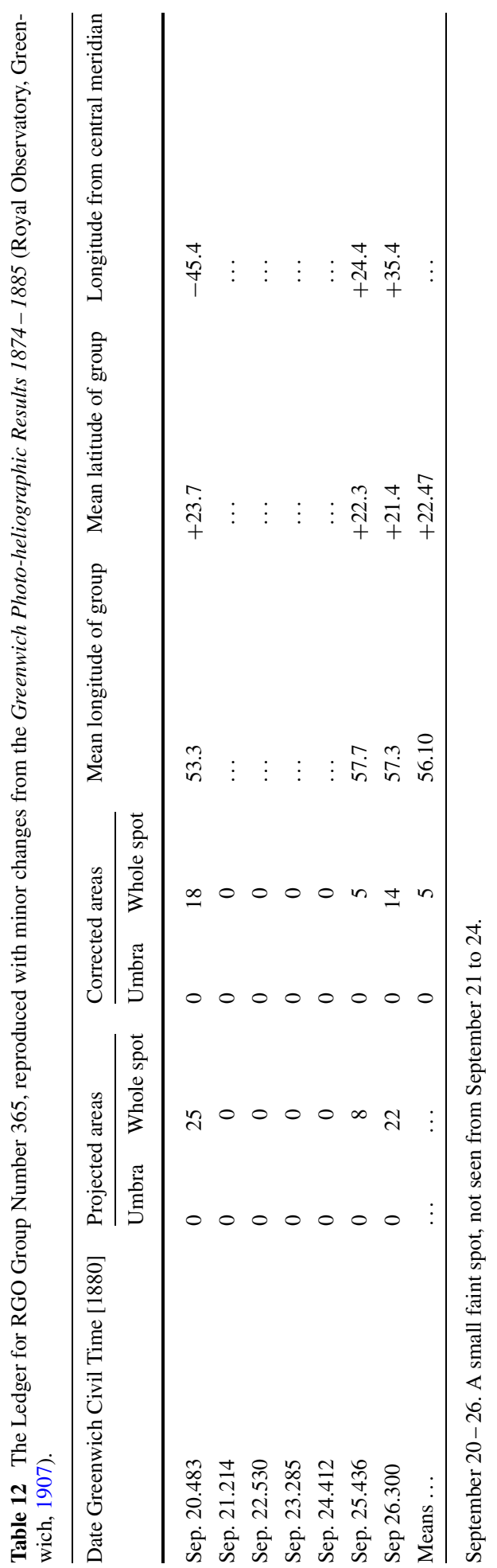


Table 13 Number of daily occurrences per calendar month, within the interval 17 April 1874-31 December 1885 , of a sunspot group not being seen on one or more days within a longer sequence of days of continual observation and for which no explanation is provided.

\begin{tabular}{|c|c|c|c|c|c|c|c|c|c|c|c|c|c|}
\hline \multirow[t]{2}{*}{ Year } & \multicolumn{12}{|c|}{ Month } & \multirow[t]{2}{*}{ Total } \\
\hline & Jan & Feb & Mar & Apr & May & Jun & Jul & Aug & Sep & Oct & Nov & Dec & \\
\hline 1874 & - & - & - & 0 & 0 & 0 & 0 & 0 & 0 & 0 & 0 & 0 & 0 \\
\hline 1875 & 0 & 0 & 1 & 0 & 0 & 0 & 0 & 0 & 0 & 0 & 0 & 0 & 1 \\
\hline 1876 & 0 & 0 & 0 & 0 & 0 & 0 & 1 & 0 & 0 & 0 & 0 & 0 & 1 \\
\hline 1877 & 0 & 0 & 0 & 0 & 0 & 0 & 0 & 0 & 0 & 0 & 0 & 0 & 0 \\
\hline 1878 & 0 & 0 & 0 & 0 & 0 & 0 & 0 & 0 & 0 & 0 & 0 & 0 & 0 \\
\hline 1879 & 0 & 0 & 0 & 0 & 0 & 0 & 0 & 0 & 0 & 0 & 0 & 0 & 0 \\
\hline 1880 & 0 & 0 & 0 & 0 & 0 & 1 & 0 & 0 & 11 & 4 & 5 & 0 & 21 \\
\hline 1881 & 0 & 0 & 2 & 10 & 1 & 1 & 2 & 1 & 0 & 1 & 2 & 4 & 24 \\
\hline 1882 & 0 & 1 & 3 & 1 & 0 & 0 & 3 & 0 & 0 & 2 & 1 & 0 & 11 \\
\hline 1883 & 0 & 0 & 0 & 0 & 0 & 0 & 0 & 0 & 0 & 1 & 0 & 1 & 2 \\
\hline 1884 & 0 & 0 & 0 & 0 & 0 & 0 & 0 & 0 & 1 & 2 & 0 & 1 & 4 \\
\hline 1885 & 0 & 0 & 6 & 1 & 1 & 0 & 0 & 0 & 0 & 0 & 3 & 1 & 12 \\
\hline Total & 0 & 1 & 12 & 12 & 2 & 2 & 6 & 1 & 12 & 10 & 11 & 7 & 76 \\
\hline
\end{tabular}

Conversely, in a few cases an explanation is given for a sunspot group being invisible on a certain day. For example, the Ledgers indicate that Group Number $150 \dagger$ apparently existed on the solar disk throughout the interval 17-20 March 1875, but had zero umbral and whole-spot areas on 18 March. The verbal description before the table reads as follows: "A small spot, not seen on March 18; probably hidden by the wire." Therefore, an explanation is given for Group Number $150 \dagger$ being invisible on 18 March. Similarly, the Ledgers indicate that Group Number 174 apparently existed on the solar disk throughout the interval 29 September-2 October 1875, but had zero umbral and whole-spot areas on 1 October. However, the verbal description before the table reads as follows: "One small spot. The photograph on October 1 is too dense for the group to be seen." Hence an explanation is given for Group Number 174 being invisible on 1 October, in the sense that the expression "too dense" presumably means that the photograph was overexposed.

The policy adopted in this investigation is to assume that if a sunspot group is not seen on a certain day (or days), and no explicit explanation is given, then that group does not contribute to the RGO count of the total number of groups on the day for which it had zero umbral and whole-spot areas. Conversely, if an explanation is given, then the sunspot group does contribute to the count of the total number of groups on the day for which it apparently had zero umbral and whole-spot areas. Table 13 presents the number of daily occurrences per calendar month, within the interval 17 April 1874-31 December 1885, of a sunspot group not being seen on one or more days within a longer sequence of days of continual observation and for which no plausible explanation is presented.

For completeness, the RGO numbers of the sunspot groups in the interval 17 April $1874-$ 31 December 1885 that were not seen on one or more days within a longer sequence of days of continual observation are listed in Appendix B. Section B.2 lists those cases where no explanation is given; Section B.3 lists those cases where a plausible explanation is provided.

Finally, it should be noted that examples of a sunspot group not being seen on one or more days within a longer sequence of days of continual observation are not confined to the 
interval $1874-1885$. Prompted by an insightful comment received from G.M. Appleby (Private communication, 2015), who worked in the Solar Department at the Royal Greenwich Observatory, Herstmonceux, during the period 1968 - 1973, a careful search has been made for similar examples occurring in the later stages of the programme of sunspot observations conducted under the aegis of the RGO. Several such examples have been found, but it probably suffices to record here two specific examples in the final interval 1972-1976. The RGO Notes on Sunspot Groups (Royal Greenwich Observatory, 1980) indicate that Group Number 23239 existed for the interval 30 September-8 October 1972 and include the following description: "A single spot on Sep 30; two spots, almost in contact, on Oct 1. Not seen again until Oct 8 when a tiny spot is visible." Similarly, the RGO Notes on Sunspot Groups indicate that Group Number 23525 existed for the interval 6-11 June 1974 and include the following description: "Several variable spots, not seen on Jun 7." More casual perusal of all of the RGO published information indicates that examples of a sunspot group not being seen $(\mathrm{U}=\mathrm{W}=0$, to the accuracy of the measurements) on one or more days within a longer sequence of days of continual observation occur throughout the interval 17 April 1874-31 December 1976.

\section{Discussion}

As noted in the Introduction, the main purpose of the present article is to re-examine the RGO number of sunspot groups on the solar disk for each day in the interval 17 April $1874-$ 31 December 1885. A concomitant goal of this endeavour is the derivation of a new dataset that achieves complete consistency between the three main sections of the Greenwich Photoheliographic Results 1874-1885 (Royal Observatory, Greenwich, 1907) in specifying the RGO count of the number of sunspot groups; namely i) the "Measures"; ii) the "Ledgers"; and iii) the "Total (Projected) Areas" (see Section 3.1). The third main section ("Total Areas") is valuable because it provides a clear identification of the days for which photographs were not acquired. Deriving this new dataset also involves implementing the Errata and Additions listed in the Greenwich Photo-heliographic Results 1874-1885 (Royal Observatory, Greenwich, 1907). The RGO count of the number of sunspot groups listed in the new dataset is then compared with the corresponding information published by Hoyt and Schatten (1998a, 1998b).

It is clear from Tables 9-13 that the relative importance of the different factors that result in discrepancies between this investigation and the one published by Hoyt and Schatten (1998a, 1998b) - in decreasing order of importance - are i) days without photographs, for which no true sunspot measurements are available (Table 9); ii) unobserved sunspot groups that could potentially have existed for just a single day on the occasion of a missing photograph (inferred from Tables 9 and 10); and iii) sunspot groups that are apparently invisible for one or more days within a longer sequence of days of continual observation, and for which no explanation is given (Tables 11,12 , and 13).

Figure 2 illustrates the number of days per calendar month, within the interval 17 April 1874-31 December 1885, for which solar photographs were acquired by the Royal Observatory, Greenwich. The solid-blue line in Figure 2 is a graphical representation of the information in the new dataset derived in this investigation and the broken-red line is the corresponding information extracted from the work of Hoyt and Schatten (1998a, 1998b). Table 9 provides the number of days per calendar month, within the same interval, for which solar photographs were not acquired, according to the present investigation. As a further check on the consistency of the new dataset, the addition of the monthly numbers depicted 
in Figure 2 (the solid blue line) and those presented in Table 9 yields the correct number of days in the appropriate calendar month. It is clear from Figure 2 that there are many days without photographs in the interval 1874-1885, particularly during the initial four-year interval $1874-1877$. Indeed, there are 656 days without photographs in the 12-year interval $1874-1886$ and 445 of these days occur in the first four-year interval 1874-1877. Conversely, soon after the beginning of 1875 the "raw data" published by Hoyt and Schatten (1998a, 1998b) - the broken-red line - contains "assigned" (or "interpolated") values for the count of the number of sunspot groups on the solar disk, in the sense that this count is given for every day in the calendar month, including days for which no solar photograph was acquired. Moreover, such assigned values for the number of sunspot groups on the solar disk must be uncertain because of the frequent occurrence of sunspot groups that exist for just a single day. There are 310 verifiable occurrences of an RGO sunspot group existing for just a single day in the interval $1874-1885$ and this must be an underestimate because of the large number of days (656) without photographs.

Figure 3 shows the monthly means of the RGO number of sunspot groups, and the standard errors of these monthly means, for the interval 17 April 1874-31 December 1885. As in the case of Figure 2, results presented in blue (solid error bars) are from the present investigation and results presented in red (broken candlesticks) are from the work of Hoyt and Schatten (1998a, 1998b). The histogram at the top of Figure 3 shows the number of days per calendar month without photographs according to the present investigation. It could be argued that the two sets of monthly means and standard errors presented in Figure 3 are not grossly dissimilar, although significant differences occur for some months (e.g. May 1874, December 1875 , January 1876, and July 1884). From this viewpoint, it could be claimed that the number of sunspot groups on the solar disk during the interval 17 April 1874-31 December 1885, as derived by Hoyt and Schatten (1998a, 1998b) from the RGO publications, are largely correct.

Similarly, Figure 4 shows the yearly means of the RGO number of sunspot groups, and the standard errors of these yearly means, for the interval 1874-1885. Once again, results presented in blue (solid line) are from the present investigation and results presented in red (broken line) are from the work of Hoyt and Schatten (1998a, 1998b). In the case of the yearly means, there are small but significant differences between the two sets of results for the years $1874,1875,1876,1877$, and 1884 . As already noted, there are many days in each year of the interval $1874-1877$ for which solar photographs were not acquired (see Tables 8 and 9), which is the main reason for the discrepancies shown in Figure 4. In addition, the yearly means derived in this investigation are slightly greater than those obtained from the work of Hoyt and Schatten (1998a, 1998b) throughout the interval 1882-1884, being significantly greater in 1884 . While it is definitely not claimed that the differences shown in Figures 2, 3, and 4 can be invoked to reconcile the discrepancies between the Group and International Sunspot Numbers in the interval $1874-1885$, the present re-examination of the daily number of sunspot groups for the RGO has resulted in an important new dataset. Further, this new dataset is more reliable than the one published by Hoyt and Schatten (1998a, 1998b), in the sense that no assumptions are made about the number of sunspot groups that are present on days for which no solar photograph was acquired originally.

Moreover, the differences illustrated in Figure 2 influence the calculation of Group Sunspot Number $\left[R_{\mathrm{G}}\right]$ in a more subtle way. As noted in Section 2.2, the correction factor $\left[k_{i}^{\prime}\right]$ for each individual observer is formed by dividing the total number of sunspot groups seen by the RGO "standard observer" by the total number of sunspot groups seen by the comparison observer, limiting the ratio to those days for which both observers saw one or more sunspots. The assumption made in this investigation that no true information on the 


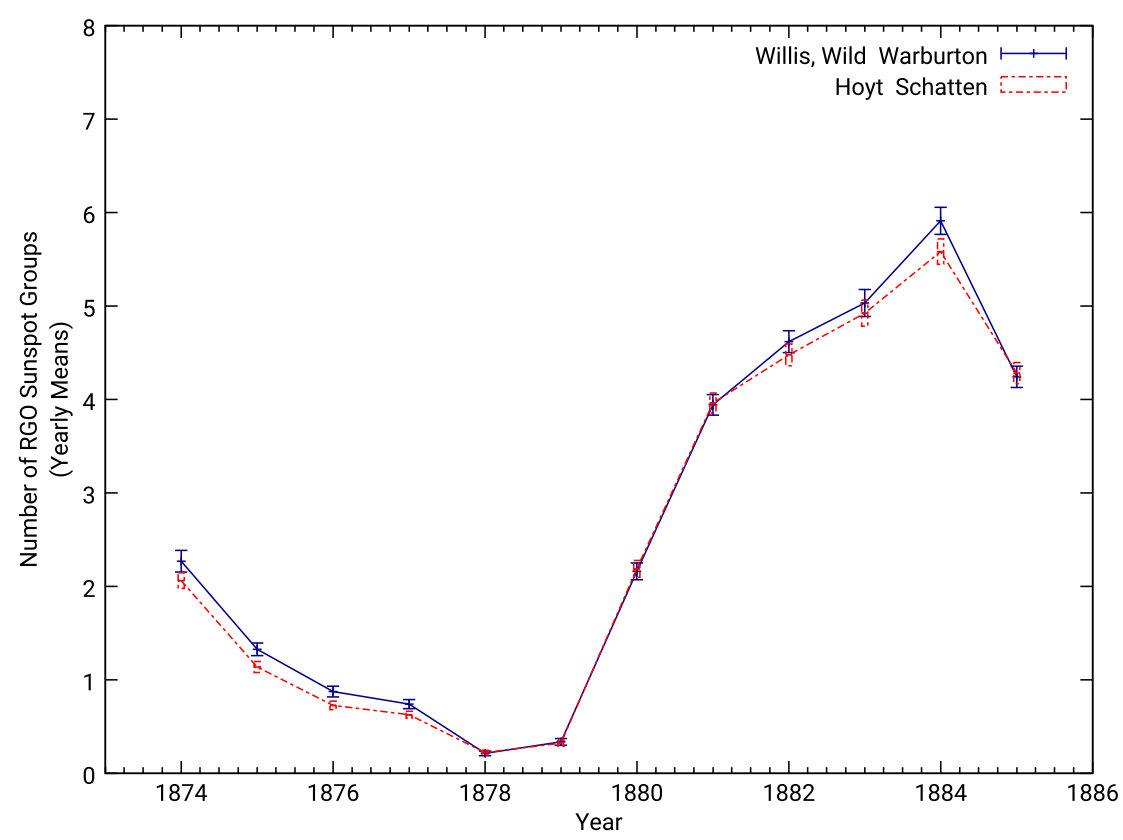

Figure 4 The yearly means of the RGO number of sunspot groups and the standard errors of these yearly means for the interval 1874-1885. As in the case of Figures 2 and 3, results presented in blue (solid line) are from the present investigation and results presented in red (broken line) are from the work of Hoyt and Schatten (1998a, 1998b).

RGO number of sunspots on the solar disk exists on days for which no photograph was acquired originally implies that the list of days for which both observers saw one or more sunspots will change. (To be slightly pedantic, the RGO "standard observer" could not have seen sunspots on days for which no photograph was available.) Therefore, the correction factor $\left[k_{i}^{\prime}\right]$ for the $i$ th comparison observer will change, at least in the sense that the arithmetical quantities change within the interval 17 April 1874-31 December 1885. Such changes, even if small individually, are insidious because they permeate through the calculation of correction factors for all observers in a way that is not entirely predictable in advance. These changes in personal correction factors result in changes to the Group Sunspot Numbers, calculated using Equation (3). Finally, it is clear from Section 2.2 that the normalisation number (12.08) will also change with the assumption that no true information on the RGO number of sunspots on the solar disk exists on days for which no photograph was acquired originally.

A possible further complication should be mentioned briefly here. One of the comparison observers listed by Hoyt and Schatten (1998a, 1998b) is Robert Main (Observer Number 334), who made sunspot observations at the Radcliffe Observatory, Oxford, during the interval 1874-1875 (Main, 1875). It is clear from the catalogue of sunspot observations published by Main (1875) that time is reckoned from Mean Noon, not Midnight. Likewise, dates in his catalogue are astronomical dates, not civil dates. In the sunspot observations published by the Royal Observatory, Greenwich, time is reckoned from Greenwich Mean Noon up until the end of 1884 but, "for convenience of reference", the month and day of the month (civil reckoning) are also given. In the (revised) Greenwich Photo-heliographic Results 1874-1885 (Royal Observatory, Greenwich, 1907) time is reckoned from Midnight 
throughout the interval $1874-1885$. Astronomical dates in the catalogue compiled by Main (1875) at times (referred to Mean Noon) between 12:00 and 23:59 become the following day in the civil calendar. These date conversions to the civil calendar must be implemented before meaningful comparisons can be made with the primary RGO "standard observer" (see Section 2.2). Such date conversions will inevitably result in a change, albeit small, to the personal correction factor $\left[k_{i}^{\prime}\right]$ for Robert Main. Similar changes would be required to the personal correction factors of all other observers using mean solar time rather than civil time.

It is important to discuss some apparent discrepancies between the results presented in this investigation and the results presented in the articles by Clette et al. (2014), Cliver et al. (2015), and Cliver and Ling (2016). The first point to be emphasised is that the present investigation only claims to revise the value of $G_{i}$, which appears on the right-hand side of Equation (3), for just the Royal Observatory, Greenwich, and for just the restricted interval 1874-1885. Moreover, the present investigation only seeks to revise the data used by Hoyt and Schatten (1998a) for the Royal Observatory, Greenwich, on the basis of the official RGO publications. Therefore, this revision relates solely to the primary RGO "standard observer" (Observer Number 332 in Hoyt and Schatten, 1998a; see their Appendix 1), whose correction factor is 1.000 by definition. The revision of the value of $G_{i}$ for the Royal Observatory, Greenwich - denoted here by $G_{332}^{*}$ - is crucially important, however, because the correction factors $\left[k_{i}\right]$ for all other observers potentially depend on $G_{332}^{*}$. Ideally, the values of $G_{i}$ for all the other 462 observers $(1 \leq i \leq 463, i \neq 332)$ listed in Appendix 1 of the article by Hoyt and Schatten (1998a) should also be checked and corrected, as necessary. Then the revised set of values $G_{i}^{*}(1 \leq i \leq 463)$ should be used to derive a revised set of correction factors $k_{i}^{*}\left(1 \leq i \leq 463, i \neq 332 ; k_{332}^{*}=1\right)$. Next, a revised normalisation number, chosen to make the mean value of the revised value of $R_{\mathrm{G}}$ equal to the mean value of $R_{\mathrm{I}}$ for the interval 1874-1976, should be calculated; this revised normalisation number would replace the existing value of 12.08. Finally, the revised values $G_{i}^{*}, k_{i}^{*}$ and the revised normalisation number should be used in Equation (3) to calculate a revised Group Sunspot Number $\left[R_{\mathrm{G}}^{*}\right]$. Only then can really accurate comparisons be made between the International $\left[R_{\mathrm{I}}\right]$ and Group $\left[R_{\mathrm{G}}^{*}\right]$ Sunspot Numbers using Equations (2) and (3).

The preceding prescriptive procedure for the ideal correction of the Group Sunspot Number $\left[R_{\mathrm{G}}\right]$ will be very difficult, if not impossible, to achieve in practice. For many observers the relevant information may no longer be available. For example, attention has already been drawn to the fact that there is apparently a mixture of astronomical and civil dates and times in the results presented by Hoyt and Schatten (1998a). In the case of sunspot observations made at the Radcliffe Observatory, Oxford, astronomical dates and times are given (Main, 1875), whereas civil dates and times are used by the Royal Observatory, Greenwich. As noted previously, some dates in the catalogue compiled by Main (Observer Number 334) become the following date (day) in the civil calendar (namely those with astronomical times between 12:00 and 23:59), which will change the personal correction factor for Main, possibly by just a small amount. Nevertheless, a revised value $G_{334}^{*}$ in the civil calendar can be derived from the information in the catalogue compiled by Main (1875) and hence, in principle, a revised value $k_{334}^{*}$ can also be calculated. Conversely, in the case of sunspot observations made at the Astronomical Observatory of Madrid (Aparicio et al., 2014), for example, it is known that civil time was used to record observations in the twentieth century: however, the time system used in the nineteenth century is unknown because the Madrid records are no longer extant and the relevant data have been retrieved from the tabulations made at the time by Wolf (J.M. Vaquero, private communication, 2015). Thus there is some uncertainty about the dates and times in the civil calendar for the sunspot observations made by Ventosa 
(Observer Numbers 336 and 343 in Hoyt and Schatten, 1998a): the actual observations were made by Ventosa, not Aguilar (336) and Merino (343), who were Directors of the Astronomical Observatory of Madrid at different times during the interval 1868-1896 (Aparicio et al., 2014). Hence the value $G_{336}$ (Madrid) is less certain than the value $G_{334}^{*}$ (Oxford) and less certain than the value $G_{332}^{*}$ (Greenwich).

In those cases for which the value of $G_{i}$ is uncertain, for whatever reason, $G_{i}$ should ideally be replaced by $G_{i} \pm \Delta G_{i}$. The determination of the appropriate value of $\Delta G_{i}$, however, is far from being immediately obvious. Perhaps the optimum situation would be to find an observer (Observer Number $j$, say) whose observations encompassed the interval $1874-1885$ (or preferably longer) and for whom the corrected value of $G_{j}$, say $G_{j}^{*}$, could be determined. Then, at least for the purposes of illustrative calculations, it might be possible to assume as a first approximation that $2 \Delta G_{i} \propto\left|G_{j}^{*}-G_{j}\right|$ for all observers for whom a corrected value of $G_{i}$ cannot be derived, although the corresponding constants of proportionality would still have to be determined. Furthermore, the replacement of $G_{i}$ by $G_{i} \pm \Delta G_{i}$ inevitably leads to the replacement of $k_{i}$ by $k_{i} \pm \Delta k_{i}$. Inserting the appropriate values of $G_{i} \pm \Delta G_{i}, k_{i} \pm \Delta k_{i}$ and a revised normalisation number with its own uncertainty (which replaces 12.08) into Equation (3) and performing the arithmetical computations would yield a corrected Group Sunspot Number of the form $R_{\mathrm{G}}^{*} \pm \Delta R_{\mathrm{G}}^{*}$. The second point to be emphasised is that the quantity $\Delta R_{G}^{*}$ has never been estimated, and hence some caution should be exercised when comparing annual averages of the "uncorrected" Group Sunspot Number $\left[R_{\mathrm{G}}\right]$ with annual averages of the International Sunspot Number $\left[R_{\mathrm{I}}\right]$.

Of far greater concern, however, are the seemingly significant differences between the values of $G_{i}$ for different observers. The uncertainties involved in the complex arithmetical calculations and summations embodied in Equation (3) cannot be invoked to explain such discrepancies. For example, Figure 3 in the article by Cliver et al. (2015) apparently shows the ratio of the mean annual RGO sunspot group counts to those of Tacchini $(i=328)$, Wolfer $(i=338)$, Winkler $(i=341)$, Quimby $(i=352)$, Catania $(i=362)$, and Guillaume $(i=386)$. The second-order fit to all of the points for these six observers does not stabilise (around 1.2) until about 1915 and is only about 0.7 at the beginning (1874) of the time interval considered. A different version of Figure 3 in the article by Cliver et al. (2015) is presented in the article by Willis et al. (2016) and there are clearly some discrepancies between the two figures, particularly with respect to the size of certain calculated ratios. Any required revision of Figure 3 in the article by Cliver et al. (2015) could have potential implications for the validity of the criticisms of the RGO count of the number of sunspot groups in the early years.

Consequently, in order to perform one rigorous comparison on a daily (not annual) basis, the revised series $G_{332}^{*}$ (Greenwich) and $G_{334}^{*}$ (Oxford) have been used. There are 122 days in the interval November 1874 - December 1875, for which counts of the number of sunspot groups (on the same day in the civil calendar) are available from the Royal Observatory, Greenwich, and the Radcliffe Observatory, Oxford. The counts at these two observatories are identical on 85 days, differ by 1 on 36 days and differ by 2 on just 1 day $\left(G_{332}^{*}=4\right.$, $G_{334}^{*}=2$ on 18 March 1875). There are no days for which the difference in the counts at the two observatories exceeds 2 . The count was recorded as being zero on 26 out of the 85 days for which the counts were identical at both observatories. Of the 37 days on which the difference in the count at the two observatories was either 1 or $2, G_{332}^{*}>G_{334}^{*}$ on 18 days and $G_{332}^{*}<G_{334}^{*}$ on 19 days. On the basis of these preliminary results, it appears that there is an appreciable level of agreement (70\%) between the sunspot observations at Greenwich and Oxford. The third point to be emphasised is that this comparison between the sunspot observations at Greenwich and Oxford has been performed on a daily basis, albeit for a 
relatively short interval of time. More comprehensive and detailed comparisons between the sunspot observations at the Royal Observatory, Greenwich, and those at the Radcliffe Observatory, Oxford, are beyond the intended scope of the present article.

\section{Conclusions}

The daily number of sunspot groups on the solar disk $\left(G_{\mathrm{RGO}}=G_{332}\right)$, as determined by the programme of sunspot observations conducted under the aegis of the Royal Observatory, Greenwich, and subsequently the Royal Greenwich Observatory (RGO), has been reexamined for the interval 1874-1885. The motivation for such a re-examination is the key role that the RGO number of sunspot groups plays in the calculation of Group Sunspot Number $\left[R_{\mathrm{G}}\right]$ in the procedure adopted by Hoyt and Schatten (Hoyt, Schatten, and Nesmes-Ribes, 1994; Hoyt and Schatten 1998a, 1998b).

It is convenient to summarise succinctly in this concluding section some of the deficiencies and limitations of the procedures employed by Hoyt and Schatten (1998a, 1998b). In particular, the main changes and improvements advocated as a result of the present study may be listed as follows:

i) Days for which no solar photograph was acquired originally by the RGO should be regarded, without exception, as days for which no true sunspot observations are available. Some of the values that Hoyt and Schatten assign to the number of sunspot groups on the solar disk on days without photographs are clearly lower limits. Moreover, in the absence of a photograph, the (unmeasured) number of sunspot groups is always uncertain because of the known frequent occurrence of sunspot groups that exist for just a single day.

ii) An agreed policy should be adopted in the case of sunspot groups that are apparently invisible for one or more days within a longer sequence of days of continual observation, in the sense that the umbral and whole-spot areas are both recorded as being zero in the RGO printed publications. The preliminary policy adopted in this investigation is to assume that if a particular sunspot group was not seen on a certain day (or sequence of days), and no explanation is given, that group does not contribute to the count of the total number of groups on days for which it had zero umbral and whole-spot areas. Conversely, if an explanation is given, that sunspot group does contribute to the total count.

iii) Similarly, a clear policy should be adopted over the specification of dates and times. The apparent use of both astronomical and civil dates and times by Hoyt and Schatten introduces an added complication in the calculation of Group Sunspot Numbers. The preliminary policy adopted in this investigation is to use civil dates and times (reckoned from Midnight), wherever possible, since this system is used for the Greenwich sunspot observations.

iv) The exclusion of days without photographs, and also days for which the umbral and whole-spot areas are both zero, inevitably changes the list of days for which comparisons can be made between the primary RGO (standard) observer and the secondary (comparison) observer. Similarly, the conversion of some astronomical dates to civil dates will produce some alterations in the list of days for which such comparisons can be made. This revision of the days for which comparisons are made, starting with direct comparisons made during the interval $1874-1885$, changes the personal correction factors of all secondary observers. These changes then influence the determination of the personal correction factors for tertiary observers, which in turn influence the correction factors for fourth-, fifth-, sixth-, and seventh-level observers. These changes in personal correction factors, even if small, are insidious and propagate through the arithmetical calculations defined by Equation (3) in a largely unpredictable manner. 
v) The calculation of personal correction factors is yet further complicated by the fact that Cliver and Ling (2016) found that they could reproduce the personal correction factors $\left[k^{\prime}\right]$ for the solar observers listed by Hoyt and Schatten (1998a: see their Appendix 1) only if they used the interpolated filldata files of observer matrices from the NOAA National Geophysical Data Center website, rather than the un-interpolated alldata files of observer matrices. Therefore, the personal correction factors published by Hoyt and Schatten (1998a) should be treated with considerable caution.

vi) The normalisation number (12.08) in Equation (3) also changes as a result of the various changes in the personal correction factors of comparison observers.

vii) If meaningful comparisons are to be made between the Group Sunspot Number and the International Sunspot Number, an attempt should be made to determine the numerical uncertainties in the Group Sunspot Number. If possible, a similar attempt should be made to determine the corresponding numerical uncertainties in the International Sunspot Number.

viii) Comprehensive comparisons between the sunspot photographs acquired by the Royal Observatory, Greenwich, and the contemporaneous sunspot drawings made at the Radcliffe Observatory, Oxford, should be undertaken.

ix) It remains to consider the effects of the different observing telescopes and photographic techniques used at the various solar observatories contributing to the RGO programme of sunspot observations on the determination of the RGO number of sunspot groups on the solar disk. These important matters are considered in the companion article by Willis et al. (2016).

Although the preceding conclusions and associated recommendations necessarily imply the need for further significant studies in the future, it is hoped that the new dataset for the daily number of sunspot groups on the solar disk for the RGO, which is derived here for the restricted interval $1874-1885$, will be a valuable initial contribution to such an endeavour.

Acknowledgements The authors thank G.M. Appleby, R.A. Harrison, A.J. Perkins, and J.M. Vaquero for much valuable advice and assistance during the preparation of this article. They also thank the referee for the constructive comments and suggestions that helped to improve the article. J.S. Warburton made an important contribution to the project while working at the Rutherford Appleton Laboratory as a summer vacation student from Imperial College London. The sterling efforts of many individuals have contributed to the publication of the Greenwich Photo-heliographic Results, 1874-1976. Tables 11 and 12 contain public sector information licensed under the Open Government Licence v2.0. The authors are indebted to staff at the Cambridge University Library, UK, for making the catalogue of solar plates and contact prints available online, and for providing specialist help in the preparation of this article. Figure 1 is reproduced by kind permission of the Syndics of Cambridge University Library.

Disclosure of Potential Conflicts of Interest The authors declare that they have no conflicts of interest.

Open Access This article is distributed under the terms of the Creative Commons Attribution 4.0 International License (http://creativecommons.org/licenses/by/4.0/), which permits unrestricted use, distribution, and reproduction in any medium, provided you give appropriate credit to the original author(s) and the source, provide a link to the Creative Commons license, and indicate if changes were made.

\section{Appendix A: Sunspot Groups that Existed for a Single Day}

\section{A.1 Aim of the Appendix}

The aim of this appendix is to list all of the sunspot groups within the interval 17 April $1874-31$ December 1885 that were seen on one day only. As noted in Section 4.3, to define rigorously a sunspot group that was seen on just one day, it must be absent on photographs 
acquired on both the immediately preceding and following days. Therefore, those sunspot groups in the "Ledgers" that have an entry for a single date are not included in the present list if no photograph was acquired on either the preceding day or the following day. As a further refinement in the future, proper allowance should be made for any sunspot group that was so close to either the east or west limb of the Sun when a photograph was taken that it could not possibly be seen, respectively, on photographs taken on either the preceding or following day, because the sunspot group would not then be on the visible solar disk. However, by examining the recorded longitude of each sunspot group from the central meridian at the time the relevant photograph was taken, it can readily be verified that relatively few of the sunspot groups listed in Section A.2 would be eliminated in such a future refinement.

\section{A.2 List of Sunspot Groups Seen on a Single Day}

The following list gives the RGO number of each sunspot group seen for one day only, together with the single date of its occurrence, for the years 1874 to 1885 .

1874: None.

1875: 143* (26 Jan); 149† (12 Mar); 157† (22 May); 162* (21 Jun); $164 \dagger(17$ Jul); 169 (30 Aug); 172 (15 Sep).

1876: 206 (4 Apr); 206* (6 Apr); 208† (13 Apr); 230 (19 Nov).

1877: $258^{*}$ (25 Sep).

1878: 268A (26 Feb); 268B (27 Feb); 271* (5 Apr); 274 (10 Jun); 276 (26 Jul).

1879: 280 (18 Apr); 293 (7 Nov).

1880: 301C (10 Jan); 304 (12 Jan); 306A (24 Jan); 307 (26 Jan); 312A (22 Feb); 317* (1 Apr); 321A (5 May); 322 (7 May); 325A (24 May); 335 (29 Jun); 337 (13 Jul); 343A (8 Aug); 343B (8 Aug); 350 (28 Aug); 359A (9 Sep); 371 (1 Oct); 376 (7 Oct); 377 (8 Oct); 379 (12 Oct); 380 (16 Oct); 382 (18 Oct); 382D (27 Oct); 382F (29 Oct); 383A (1 Nov); 383B (1 Nov); 388 (4 Nov); 389 (8 Nov); 390 (9 Nov); 391 (9 Nov); 392A (14 Nov); 399 (27 Nov).

1881: 407A (13 Jan); 410C (20 Jan); 420 (9 Feb); 421 (9 Feb); 424B (18 Feb); 427A (27 Feb); 427B (27 Feb); 438 (18 Mar); 440 (19 Mar); 440A (20 Mar); 440B (21 Mar); 441 (22 Mar); 444 (29 Mar); 446 (1 Apr); 449 (2 Apr); 457A (17 Apr); 458* (18 Apr); 462A (21 Apr); 465* (26 Apr); 466A (1 May); 468 (5 May); 472A (10 May); 473 (13 May); 476 (19 May); 479 (21 May); 483 (30 May); 486 (31 May); 495A (15 Jun); 499 (23 Jun); 512 (5 Jul); 513 (5 Jul); 519 (13 Jul); 528A (24 Jul); 530 (28 Jul); 544 (10 Aug); 545 (11 Aug); 551 (23 Aug); 554A (25 Aug); 555 (26 Aug); 564 (6 Sep); 575A (22 Sep); 586A (9 Oct); 595A (27 Oct); 596 (30 Oct); 596A (1 Nov); 597 (5 Nov); 601A (7 Nov); 608 (16 Nov); 610 (21 Nov); 611 (21 Nov); 612 (23 Nov); 615 (28 Nov); 617 (29 Nov); 624 (14 Dec); 629A (13 Dec); 633 (23 Dec); 635 (28 Dec); 636 (30 Dec).

1882: 640 (4 Jan); 644 (6 Jan); 663 (2 Feb); 673 (13 Feb); 674 (13 Feb); 679 (16 Feb); 680 (18 Feb); 681 (18 Feb); 682 (18 Feb); 686 (27 Feb); 688 (28 Feb); 689* (4 Mar); 696 (9 Mar); 698 (10 Mar); 700 (13 Mar); 706 (22 Mar); 712 (27 Mar); 719 (4 Apr); 724 (8 Apr); 727 (11 Apr); 730 (14 Apr); 745 (2 May); 748 (3 May); 751 (5 May); 758 (10 May); 761 (13 May); 764 (20 May); 768 (1 Jun); 769 (3 Jun); 772 (3 Jun); 773 (4 Jun); 775 (7 Jun); 776 (7 Jun); 784 (24 Jun); 799 (17 Jul); 801 (18 Jul); 809 (3 Aug); 813 (10 Aug); 825 (30 Aug); 832 (2 Sep); 834 (2 Sep); 867 (22 Oct); 879 (8 Nov); 881 (11 Nov); 886 (14 Nov); 901 (30 Nov); 902 (2 Dec); 905 (7 Dec); 915 (21 Dec); 917 (22 Dec); 919 (26 Dec); 922 (28 Dec).

1883: 932 (10 Jan); 935 (13 Jan); 937 (16 Jan); 947 (28 Jan); 950 (30 Jan); 951 (30 Jan); 952 (30 Jan); 953 (30 Jan); 957 (2 Feb); 958 (6 Feb); 965 (13 Feb); 966 (13 Feb); 968 
(16 Feb); 970 (19 Feb); 983 (22 Mar); 993 (2 Apr); 995 (6 Apr); 1005 (22 Apr); 1006 (22 Apr); 1019 (3 May); 1020 (3 May); 1023 (6 May); 1024 (7 May); 1029 (12 May); 1031 (15 May); 1034 (19 May); 1035 (23 May); 1038 (29 May); 1047 (7 Jun); 1050 (13 Jun); 1055 (19 Jun); 1057 (21 Jun); 1059 (24 Jun); 1060 (25 Jun); 1063 (27 Jun); 1069 (11 Jul); 1081 (20 Jul); 1082 (20 Jul); 1096 (11 Aug); 1097 (14 Aug); 1113 (4 Sep); 1124 (22 Sep); 1125 (22 Sep); 1143 (12 Oct); 1145* (14 Oct); 1151 (21 Oct); 1159 (28 Oct); 1167 (2 Nov); 1175 (13 Nov); 1178 (16 Nov); 1189 (25 Nov); 1203 (10 Dec); 1207 (13 Dec); 1210 (17 Dec); 1222 (28 Dec).

1884: 1238 (6 Jan); 1239 (6 Jan); 1242 (7 Jan); 1245 (8 Jan); 1248 (10 Jan); 1257 (19 Jan); 1258 (22 Jan); 1274 (8 Feb); 1275 (8 Feb); 1316 (10 Mar); 1318 (11 Mar); 1328 (18 Mar); 1329 (18 Mar); 1332 (20 Mar); 1333 (20 Mar); 1334 (21 Mar); 1335 (21 Mar); 1338 (24 Mar); 1345 (1 Apr); 1349 (3 Apr); 1361 (14 Apr); 1364 (15 Apr); 1365 (16 Apr); 1367 (18 Apr); 1386 (12 May); 1399 (25 May); 1401 (29 May); 1402 (29 May); 1413 (14 Jun); 1428 (9 Jul); 1429 (9 Jul); 1447 (2 Aug); 1449 (6 Aug); 1464* (25 Aug); 1472 (3 Sep); 1473 (4 Sep); 1478 (6 Sep); 1494 (23 Sep); 1507 (15 Oct); 1510 (16 Oct); 1511 (18 Oct); 1512 (18 Oct); 1517 (21 Oct); 1527 (1 Nov); 1529 (1 Nov); 1531 (2 Nov); 1540 (13 Nov); 1562 (25 Dec); 1565 (30 Dec).

1885: 1568 (2 Jan); 1577 (22 Jan); 1583 (29 Jan); 1603 (18 Feb); 1605 (20 Feb); 1611 (25 Feb); 1616 (2 Mar); 1628 (19 Mar); 1633 (28 Mar); 1635 (28 Mar); 1640 (10 Apr); 1644 (19 Apr); 1648 (25 Apr); 1654 (28 Apr); 1655 (28 Apr); 1657 (1 May); 1660 (1 May); 1667 (8 May); 1668 (9 May); 1680 (21 May); 1691 (3 Jun); 1696 (9 Jun); 1698 (12 Jun); 1721 (14 Jul); 1723 (16 Jul); 1723* (17 Jul); 1734 (28 Jul); 1739 (7 Aug); 1741 (8 Aug); 1743 (9 Aug); 1749 (16 Aug); 1752 (19 Aug); 1753 (20 Aug); 1761 (5 Sep); 1762 (5 Sep); 1772 (28 Sep); 1781 (18 Oct); 1785 (23 Oct); 1799 (23 Nov); 1800 (26 Nov); 1804 (9 Dec); 1805 (9 Dec); 1809 (14 Dec); 1811 (19 Dec); 1813* (21 Dec); 1818 (25 Dec).

\section{Appendix B: Sunspot Groups that were Temporarily Invisible}

\section{B.1 Aim of the Appendix}

The aim of this appendix is to list all of the sunspot groups in the interval 17 April $1874-$ 31 December 1885 that had zero umbral (U) and whole-spot (W) areas for one or more days within a longer interval of continual observation, during which $\mathrm{U}$ and $\mathrm{W}$ were not both zero. As noted in Section 4.4, in most cases no explanation is given for zero umbral and whole spot areas but in a few cases a plausible explanation is provided. Therefore, separate lists are presented depending on whether or not an explanation is given.

\section{B.2 List of Temporarily Invisible Sunspot Groups: No Explanation Given}

The following list gives the RGO number of each sunspot group that was not seen for one or more days, together with the date range of its invisibility, for the years 1874 to 1885 . No explanation is given in the RGO publications for the temporary invisibility of these groups.

1874: None.

1875: $149^{*}$ (15 Mar).

1876: 217 (29 Jul).

1877-1879: None.

1880: 333* (29 Jun); 356 (8 - 9 Sep); 358 (12 Sep); 361 (25 - 26 Sep); 365 (21 - 24 Sep); 366 (26 Sep); 367 (26 Sep); 373 (9 Oct); 374 (10 Oct); 380† (17-18 Oct); 395 (20 Nov, $22-24$ Nov, and 26 Nov). 
1881: 434 (12-13 Mar); 454 (10-17 Apr); 458 (19-20 Apr); 470 (6 May); 495 (22 Jun); 518 (17 Jul); 523 (20 Jul); 542 (10 Aug); 579 (2 Oct); 601 (15 Nov); 613 (30 Nov and $1-2 \mathrm{Dec}) ; 622$ (13 Dec); 631 (24 Dec).

1882: 683 (19 Feb); 710 (26 - 28 Mar); 731 (17 Apr); 793 (2 Jul); 795 (13 - 14 Jul); 865

(24 Oct); 871 (31 Oct and 1 Nov).

1883: 1153 (24 Oct); 1204 (15 Dec).

1884: 1487 (19 Sep); 1514 (22 Oct); 1515 (22 Oct); 1564 (30 Dec).

1885: 1619 (8 Mar); 1623 (14-16 Mar); 1625 (15 and 17 Mar); 1637 (5 Apr); 1685 (31 May); 1789 (5 Nov); 1791 (8 Nov); 1797 (25 Nov); 1814 (24 Dec).

\section{B.3 List of Temporarily Invisible Sunspot Groups: Plausible Explanation Presented}

The following list gives the RGO number of each sunspot group that was not seen for one or more days, together with the date range of its invisibility, for the years 1874 to 1885 . A plausible explanation is provided in the RGO publications for the temporary invisibility of these groups and the relevant text is included after the cited date.

1874: None.

1875: $150 \dagger$ (18 Mar: "A small spot, not seen on March 18; probably hidden by the wire."); 174 (1 Oct: "One small spot. The photograph on October 1 is too dense for the group to be seen.”).

1876-1879: None.

1880: 403 (31 Dec: "A large regular spot. It is eclipsed on the photograph taken on December 31 during the Solar Eclipse.”).

1881 - 1885: None.

\section{References}

Aparicio, A.J.P., Vaquero, J.M., Carrasco, V.M.S., Gallego, M.C.: 2014, Sunspot numbers and areas from the Madrid Astronomical Observatory (1876-1986). Solar Phys. 289, 4335. DOI.

Barnard, L., Lockwood, M., Hapgood, M.A., Owens, M.J., Davis, C.J., Steinhilber, F.: 2011, Predicting space climate change. Geophys. Res. Lett. 38, L16103. DOI.

Charbonneau, P.: 2010, Dynamo models of the solar cycle. Living Rev. Solar Phys. 7, 3. DOI. www. livingreviews.org//rsp-2010-3.

Clette, F., Berghmans, D., Vanlommel, P., Van der Linden, R.A.M., Koeckelenbergh, A., Wauters, L.: 2007, From the Wolf number to the International Sunspot Index: 25 years of SIDC. Adv. Space Res. 40, 919.

Clette, F., Svalgaard, L., Vaquero, J.M., Cliver, E.W.: 2014, Revisiting the sunspot number: A 400-year perspective on the solar cycle. Space Sci. Rev. 186, 35. DOI.

Cliver, E.W., Ling, A.G.: 2016, The discontinuity circa 1885 in the group sunspot number. Solar Phys., 1. DOI.

Cliver, E.W., Clette, F., Svalgaard, L.: 2013, Recalibrating the Sunspot Number (SSN): The SSN Workshops. Cent. Eur. Astrophys. Bull. 37, 401.

Cliver, E.W., Clette, F., Svalgaard, L., Vaquero, J.M.: 2015, Recalibrating the Sunspot Number (SN): The 3rd and 4th SN Workshops. Cent. Eur. Astrophys. Bull. 39, 1.

Erwin, E.H., Coffey, H.E., Denig, W.F., Willis, D.M., Henwood, R., Wild, M.N.: 2013, The Greenwich Photoheliographic Results (1874-1976): Initial corrections to the printed publications. Solar Phys. 288, 157. DOI.

Gray, L.J., Beer, J., Geller, M., Haigh, J.D., Lockwood, M., Matthes, K., Cubasch, U., Fleitmann, D., Harrison, G., Hood, L., Luterbacher, J., Meehl, G.A., Shindell, D., van Geel, B., White, W.: 2010, Solar influences on climate. Rev. Geophys. 48, RG4001. DOI.

Greenwich Astronomical Results: 1874, Results of the Astronomical Observations Made at the Royal Observatory, Greenwich, 1874 (Extracted from the Greenwich Observations, 1874), HM Stationery Office, London.

Hoyt, D.V., Schatten, K.H.: 1998a, Group Sunspot Numbers: A new solar activity reconstruction. Solar Phys. 179, 189. DOI. 
Hoyt, D.V., Schatten, K.H.: 1998b, Group Sunspot Numbers: A new solar activity reconstruction. Solar Phys. 181, 491. DOI.

Hoyt, D.V., Schatten, K.H., Nesmes-Ribes, E.: 1994, The one hundredth year of Rudolf Wolf's death: Do we have the correct reconstruction of solar activity? Geophys. Res. Lett. 21, 2067.

Lockwood, M.: 2012, Solar influence on global and regional climates. Surv. Geophys. 33(3), 503. DOI.

Lockwood, M.: 2013, Reconstruction and prediction of variations in the open solar magnetic flux and interplanetary conditions. Living Rev. Solar Phys. 10, 4. DOI. www.livingreviews.org/lrsp-2013-4.

Main, R.: 1875, Observations and delineations of solar spots made with the heliometer in the years $1874-5$. In: Results of Astronomical and Meteorological Observations made at the Radcliffe Observatory 35, 217.

McKinnon, J.A.: 1987, Sunspot numbers: 1610 - 1985. Report UAG-95, World Data Center A for SolarTerrestrial Physics, Boulder, Colorado.

Pulkkinen, T.: 2007, Space weather: Terrestrial perspective. Living Rev. Solar Phys. 4, 1. DOI. www. livingreviews.org//rsp-2007-1.

Royal Greenwich Observatory: 1980, Royal Observatory Annals Number 13 (Photoheliographic Results 1972 - 1976, Royal Greenwich Observatory, Herstmonceux), Science Research Council, Whitstable Litho Ltd, Whitstable, Kent.

Royal Observatory, Greenwich: 1907, Photo-heliographic Results 1874 to 1885, Being Supplementary Results from Photographs of the Sun Taken at Greenwich, at Harvard College, USA, at Melbourne, in India, and in Mauritius in the Years 1874 to 1885: and Measured and Reduced at the Royal Observatory, Greenwich, HM Stationery Office, Edinburgh.

Solar Physics Committee: 1891, Measures of Positions and Areas of Sun Spots and Faculae on Photographs Taken at Greenwich, Dehra Dun, and Melbourne; with the Deduced Heliographic Longitudes and Latitudes, 1878 - 1881, HM Stationery Office, London.

Waldmeier, M.: 1961, The Sunspot-Activity in the Years 1610 - 1960, Schulthess \& Company AG, Zürich.

Willis, D.M., Coffey, H.E., Henwood, R., Erwin, E.H., Hoyt, D.V., Wild, M.N., Denig, W.F.: 2013, The Greenwich Photo-heliographic Results (1874-1976): Summary of the observations, applications, datasets, definitions and errors. Solar Phys. 288, 117. DOI.

Willis, D.M., Wild, M.N., Appleby, G.M., Macdonald, L.T.: 2016, The Greenwich Photo-heliographic Results (1874-1885): Observing telescopes, photographic processes, and solar images. Solar Phys., accepted. 